\title{
Hydrodynamic force and torque models for a particle moving near a wall at finite particle Reynolds numbers
}

\author{
Zhideng Zhou ${ }^{\mathrm{a}, \mathrm{b}}$, Guodong Jin ${ }^{\mathrm{a}, \mathrm{b}, *}$, Baolin Tian ${ }^{\mathrm{c}}$, Jian Ren ${ }^{\mathrm{c}}$ \\ ${ }^{a}$ LNM, Institute of Mechanics, Chinese Academy of Sciences, Beijing 100190, China \\ ${ }^{\mathrm{b}}$ School of Engineering Science, University of Chinese Academy of Sciences, Beijing 100049, China \\ ${ }^{\mathrm{c}}$ Institute of Applied Physics and Computational Mathematics, Beijing 100088, China
}

\section{A R T I C L E I N F O}

\section{Article history:}

Received 21 March 2016

Revised 7 December 2016

Accepted 3 January 2017

Available online 17 February 2017

Keywords:

Models for hydrodynamic force and torque

Finite particle Reynolds number

Near-wall effect

Particle-resolved simulation

Sub-grid scale model

Eulerian-Lagrangian simulation

\begin{abstract}
A B S T R A C T
This research work is aimed at proposing models for the hydrodynamic force and torque experienced by a spherical particle moving near a solid wall in a viscous fluid at finite particle Reynolds numbers. Conventional lubrication theory was developed based on the theory of Stokes flow around the particle at vanishing particle Reynolds number. In order to account for the effects of finite particle Reynolds number on the models for hydrodynamic force and torque near a wall, we use four types of simple motions at different particle Reynolds numbers. Using the lattice Boltzmann method and considering the moving boundary conditions, we fully resolve the flow field near the particle and obtain the models for hydrodynamic force and torque as functions of particle Reynolds number and the dimensionless gap between the particle and the wall. The resolution is up to 50 grids per particle diameter. After comparing numerical results of the coefficients with conventional results based on Stokes flow, we propose new models for hydrodynamic force and torque at different particle Reynolds numbers. It is shown that the particle Reynolds number has a significant impact on the models for hydrodynamic force and torque. Furthermore, the models are validated against general motions of a particle and available modeling results from literature. The proposed models could be used as sub-grid scale models where the flows between particle and wall can not be fully resolved, or be used in Lagrangian simulations of particle-laden flows when particles are close to a wall instead of the currently used models for an isolated particle.
\end{abstract}

(c) 2017 Elsevier Ltd. All rights reserved.

\section{Introduction}

The wall-bounded particle-fluid two-phase flows exist widely in numerous industrial and natural processes, such as the solidfluid flow in industrial pneumatic conveying (Laín and Sommerfeld, 2012), the flows in a pump, the flows in fluidized bed (Capecelatro et al., 2014; Lu et al., 2013), sediment deposition and transport in rivers (Kidanemariam et al., 2013). One of the crucial phenomena in such flows is the interaction between particles and a solid wall.

This research work is basically interested in the motion of a single spherical particle close to a solid wall in fluid flow at finite particle Reynolds numbers. It is an elemental process in the particlefluid two-phase flows and an important ingredient in treating boundary condition in numerical simulation of such flows. The particle-resolved direct numerical simulation (PR-DNS) has been emerging as a powerful research tool for particle-fluid two-phase flows. It can be used to track the motion of particles, fully resolve

\footnotetext{
* Corresponding author.

E-mail address: gdjin@Inm.imech.ac.cn (G. Jin).
}

the surrounding fluid flows and calculate the hydrodynamic forces and torques acting on each particle (Ladd, 1994b; Lucci et al., 2010; Shao et al., 2012; Uhlmann, 2008; Uhlmann and Doychev, 2014; Wang et al., 2013; 2010). To numerically simulate the wallbounded two-phase flow, it is necessary to generate grids of finitesize in the flow domain. When the gap between the particle and the wall is smaller than one or two grid sizes, neither the fluid motion in the gap nor the force and torque on the particle can be solved accurately (ten Cate et al., 2002). Under this condition, the hydrodynamic viscous force and torque, which arises from the high pressure when the interstitial fluid is squeezed out of the small space between the two close solid surfaces, should be compensated by establishing appropriate sub-grid scale models (Nguyen and Ladd, 2002; Zhang et al., 2005).

There are two types of sub-grid scale models used to compensate the hydrodynamic force and torque on the particle when the gap is less than one or two grids. The first one is the method of potential force (Ozdemir et al., 2010; Wang et al., 2008), which means that the resistance on the particle is set to the function of gap size between the particle and the wall. The second one is the method 
of lubrication force (Dance and Maxey, 2003; Portela and Oliemans, 2003). The asymptotic formulae (Dance and Maxey, 2003; Nguyen and Ladd, 2002) of lubrication force and torque can be applied to resolve the particle-wall interaction. For example, the normal force correction can be expressed as

$\mathrm{F}^{\mathrm{lub}}=-6 \pi \mu R u_{\perp}\left(\frac{1}{\varepsilon}-\frac{1}{\Delta}\right)$

where $u_{\perp}$ is the particle velocity normal to the wall and $\Delta=\delta / R$ is a prescribed dimensionless distance cutoff below which the hydrodynamical force is compensated, $\delta$ is usually set to be one or two grid sizes, $R$ is the radius of the particle, $\varepsilon \equiv h / R$ represents a small dimensionless gap, and $h$ is the gap between the particle nose and the wall, $\mu$ is the dynamical viscosity coefficient of the fluid (ten Cate et al., 2002).

However, the current models for lubrication force and torque are based on the assumption of the Stokes flow, which might not be suitable for cases at finite particle Reynolds numbers. Here, the translational particle Reynolds number is defined as

$\operatorname{Re}_{p}=\frac{\rho_{f} u d_{p}}{\mu}$

where $d_{p}=2 R$ is the diameter of particle, $u$ is the particle velocity, and $\rho_{f}$ is the density of fluid. Therefore, the objective of this work is to develop new models for the hydrodynamic force and torque at finite particle Reynolds numbers. The new models can be used in two major applications. First, they can be used as sub-grid models in PR-DNS calculations where the flow in the gap between the particle and wall can not be sufficiently resolved. They can be used in any PR-DNS approaches, such as lattice Boltzmann method (Ladd, 1994a; 1994b), immersed boundary method (Lucci et al., 2010) and fictitious particle method (Shao et al., 2012). Second, they can be used in Lagrangian simulations of particle-laden flows to replace the currently used model for an isolated particle. In current computational fluid dynamics (CFD) codes, force and torque models on the particles are not modified when they are very close to a wall. It will be shown in this work that the force and torque on a particle near a wall can be significantly different from those on an isolated particle. Therefore, the proposed new models will be useful for Lagrangian simulations of applications such as cyclone separators (Song et al., 2016).

In order to develop the models for hydrodynamic force and torque for particle motions in a fluid at low Reynolds numbers, superposition of four simple motions of two isolated spheres is usually used to analyze their lubrication forces and torques (Dance and Maxey, 2003; Rosa et al., 2011). The four simple motions consist of (i) a sphere translating normally to another sphere, (ii) two spheres translating along the direction perpendicular to the line connecting their centers, (iii) two spheres rotating around the line connecting their centers, (iv) two spheres rotating around the direction perpendicular to the line connecting their centers. For a sphere translating towards a stationary sphere, Jeffrey (1982) combined the work of Cooley and O'Neill (1969) with numerical calculations and deduced a brief formula proportional to $\varepsilon^{-1}$ for normal force acting on the moving sphere. For the other three motions, Jeffrey and Onishi (1984) extended the work of O'Neill and Majumdar (1970) and obtained asymptotic expressions of the forces and torques acting on the spheres. Above all, the formulae were obtained based on the assumption of Stokes flow. The asymptotic formulae of the lubrication force and torque for the four simple motions are suitable for two spheres of different radii. When the radius of one of the two spheres tends to be infinite, the larger sphere could be regarded as a plane wall. Then the asymptotic formulae for two particles turned into the theoretical expressions of lubrication force and torque acting on a moving sphere near a plane wall (Dance and Maxey, 2003).
The theoretical expressions of lubrication force and torque are suitable for fluid flow at vanishing particle Reynolds numbers. However, there are many practical situations related to the motion of particles in fluid flow at finite particle Reynolds numbers. As for the particles in channel flows, Uhlmann (2008) fully resolved the phase interfaces by DNS with an immersed boundary method and García-Villalba et al. (2012) simulated turbulent flow in a vertical plane channel seeded with heavy spherical particles. Uhlmann and Doychev (2014) simulated the gravity-induced motion of finite-size particles in fluid in triply periodic domains. The particle Reynolds numbers of the flows are greater than 100 . They used the repulsive force mechanism to recover the close particle-particle hydrodynamic interaction. Zeng et al. (2008; 2010) considered the turbulent channel flow over an isolated particle with variable sizes and locations. There are also pipe flows laden with particles, such as the experimental study of turbulent flow driven by particles in pipe flows (Belt et al., 2012) and the gassolid flows in wall-bounded vertical risers (Laín and Sommerfeld, 2012; Lu et al., 2013; Wang et al., 2013). In addition, Lucci et al. (2010) numerically simulated the turbulent flow around moving spherical particles dispersed in a decaying isotropic turbulent flow. Yeo et al. (2010) investigated the modulation of isotropic turbulent flows induced by spherical bubbles, neutrally buoyant particles and slightly inertial particles. They also used the repulsive force between the particle surfaces when the gap is less than a critical value. In current large-eddy simulation of particle-laden channel flows, the unresolved sub-grid scale motion might affect the particle-wall interaction (Bianco et al., 2012). The contributions of the sub-grid scale fluid motions on particle-wall interaction can be partially modeled by constructing particle sub-grid scale model based on the space-time correction theory (He et al., 2002; Yang et al., 2008; Zhao and He, 2009).

The experimental investigations and numerical simulations described above are related to particle-particle and particle-wall hydrodynamical interaction at finite particle Reynolds numbers. The typical particle Reynolds numbers are respectively listed in Table 1.

Illustrated in Table 1 is the range of particle Reynolds numbers in many situations, the range is about $O(10) \sim O(100)$. Although the local particle Reynolds number near the wall can be reduced by the hydrodynamic force, the effects of finite particle Reynolds number will have to be considered. Experimental studies show that when the particle Stokes number $S t=\tau_{p} / \tau_{f}=\left(\left(\rho_{p} / \rho_{f}\right) / 9\right) \operatorname{Re}_{p}$ is larger than a critical value, $S t^{*}=10$, the particle will approach the wall with a finite velocity and rebound back, where $\tau_{p}$ is particle relaxation timescale and $\tau_{f}$ is a characteristic timescale of the flow (Gondret et al., 2002; Joseph et al., 2001). Using the method of matched asymptotic expansions, Cox and Brenner (1967) considered the contribution of fluid inertia at a small but finite particle Reynolds number to the lubrication force by multiplying the Stokes drag force with a dimensionless friction factor $f_{z z}(\varepsilon)$,

$f_{z z}(\varepsilon)=\frac{1}{\varepsilon}+\frac{1}{5}\left(1+\frac{R e_{p}}{4}\right) \ln \left(\frac{1}{\varepsilon}\right)+O\left(R e_{p}^{2}\right)$,

where $\varepsilon \ll 1$ and $\varepsilon R e_{p} \ll 1$ and the particle approaches the wall with a constant speed. The moving conditions of a particle at constant translational or rotational speeds are also applied in this work. Liu and Prosperetti (2010) considered a sphere rotating at $R e_{\Omega} \leq 200$ near one or two infinite plane walls parallel or perpendicular to the axis of rotation and studied the centrifugal, inertial and viscous effects on the hydrodynamic force and torque acting on the sphere. Here, the rotational particle Reynolds number is defined as

$\operatorname{Re}_{\Omega}=\frac{\rho_{f} \Omega R d_{p}}{\mu}$

where $\Omega$ is the angular velocity of the sphere. Tagawa et al. (2013) investigated the wall effect on a repulsive force act- 
Table 1

Typical particle Reynolds numbers in the particle-fluid two-phase flows in literatures.

\begin{tabular}{|c|c|c|}
\hline References & Types of flows & $R e_{p}$ \\
\hline Kim and Balachandar (2012) & An isolated finite-sized particle subjected to isotropic turbulent cross-flow & $100,250,350$ \\
\hline Zeng et al. (2010) & A finite-sized stationary particle in a channel flow of modest turbulence & $40 \sim 450$ \\
\hline Lucci et al. (2010) & Finite-sized solid spherical particles in decaying isotropic turbulence & $O(10)(65 / 75 / 280)$ \\
\hline Belt et al. (2012) & Particle-laden secondary flow in turbulent pipe flows & 110,217 \\
\hline $\mathrm{Xu}$ and Bodenschatz (2008) & Particles in intense turbulent water flows & $22,35,55$ \\
\hline Kidanemariam et al. (2013) & Horizontal open channel flow with finite-size, heavy particles & $15 \sim 20$ \\
\hline Laín and Sommerfeld (2012) & Pneumatic conveying of spherical particles in horizontal ducts & 40 \\
\hline Dorgan and Loth (2004) & Particles released near the wall in a turbulent boundary layer & $10^{-5} \sim 30$ \\
\hline Zeng et al. (2008) & Turbulent channel flow over an isolated particle of finite-size & $42 \sim 295,325 / 455$ \\
\hline Tenneti and Subramaniam (2014) & Gas-solid flows & 20,50 \\
\hline Wang et al. (2008) & Sedimentation of 1,2 or 105 particles in a channel flow & about 17.3, 503 \\
\hline García-Villalba et al. (2012) & Vertical plane channel flow with finite-size particles & 132 \\
\hline Uhlmann (2008) & Vertical particulate channel flow & 136 \\
\hline Uhlmann and Doychev (2014) & $\begin{array}{l}\text { The gravity-induced motion of randomly distributed, finite-size, heavy particles } \\
\text { in quiescent fluid in triply periodic domains }\end{array}$ & $141.1,233.1,260.6$ \\
\hline Shao et al. (2012) & Particle-laden turbulent flow in a horizontal channel & $22.2,36.2$ \\
\hline Wang et al. (2010) & Particle-fluid systems & 1 \\
\hline Wang et al. (2014) & Single-phase turbulence and particle-laden turbulence & $O(10)$ \\
\hline
\end{tabular}

ing on a sphere near the wall and experimentally study translational and rotational motion of a particle slightly heavier than the fluid in a rotating drum filled with water. Lin and Lin (2013) numerically studied the effects of finite particle Reynolds numbers up to $R e_{p}=50$ on the model for normal lubrication force on a particle moving towards a solid wall using the immersed boundary method. By fitting the numerical results, they proposed a model for the normal lubrication force. In this work, the models for both forces and torques on a particle with more general motions near a solid wall will be extended, including the translational and rotational motions at a wider range of particle Reynolds numbers. The lattice Boltzmann method is used to fully resolve the flow around the moving particle and calculate the hydrodynamic force and torque on it.

The rest of paper is organized as follows: Section 2 describes the models for hydrodynamic force and torque in Stokes flow limit, and proposes new models at finite particle Reynolds numbers. The four types of simple particle motions are also described in order to obtain the coefficients of forces and torques. Section 3 introduces the numerical methods. The basic principles of the multiplerelaxation-time lattice Boltzmann method and the equations of particle motions are discussed. Section 4 shows the effects of computational domain size, grid-size convergence, comparison of current numerical results with previous studies and the coefficients of the new proposed models. The corrected formulae of the five independent coefficients of the models for hydrodynamic force and torque are described in detail. In Section 5, the conclusions and applications about the models for the hydrodynamic force and torque at finite particle Reynolds numbers are presented.

\section{Modeling hydrodynamic force and torque}

\subsection{Models for hydrodynamic force and torque in the Stokes flow limit}

For a single particle moving near a plane wall in a Stokes flow, the linear resistance relations between the hydrodynamic force and torque on the particle and the translational and angular velocities of the particle can be expressed as (Dance and Maxey, 2003)

$\left\{\begin{array}{l}F_{i}=6 \pi \mu R\left(A_{i j} U_{j}+R B_{i j} \Omega_{j}\right) \\ T_{i}=8 \pi \mu R^{2}\left(C_{i j} U_{j}+R D_{i j} \Omega_{j}\right)\end{array}\right.$

where $i$ and $j=1,2,3$ denote the $\mathrm{y}, \mathrm{x}$ and $\mathrm{z}$ direction respectively in a reference frame attached to the wall, as shown in Fig. $1, F_{i}$ denotes the force along the axis $i$ and $T_{i}$ denotes the torque about
Table 2

The coefficients of hydrodynamic force and torque when a particle moves close to a solid wall in fluid flow at vanishing particle Reynolds number. In the table, the theoretical expressions of $A_{11}, A_{22}, B_{23}, D_{33}$ are from Dance and Maxey (2003). The model for the coefficient $D_{11}$ is fitted from the result by Jeffery (1915).

\begin{tabular}{|c|c|c|c|c|c|c|}
\hline & & Theoretical expression & & $O(1)$ & & \\
\hline$A_{11}$ & $=$ & $-\frac{1}{\varepsilon}+\frac{1}{5} \ln \varepsilon+\frac{1}{21} \varepsilon \ln \varepsilon$ & + & -0.848 & + & $O(\varepsilon)$ \\
\hline$A_{22}$ & $=$ & $\frac{8}{15} \ln \varepsilon+\frac{64}{375} \varepsilon \ln \varepsilon$ & + & -0.952 & + & $O(\varepsilon)$ \\
\hline$B_{23}$ & $=$ & $-\frac{2}{15} \ln \varepsilon-\frac{86}{375} \varepsilon \ln \varepsilon$ & + & -0.257 & + & $O(\varepsilon)$ \\
\hline$D_{33}$ & $=$ & $\frac{2}{5} \ln \varepsilon+\frac{66}{125} \varepsilon \ln \varepsilon$ & + & -0.371 & + & $O(\varepsilon)$ \\
\hline$D_{11}$ & $=$ & $-1.277+0.285 \cdot \varepsilon^{0.25}$ & & & & \\
\hline
\end{tabular}

the axis $i$. A, B, C, D are dimensionless second rank tensors. The Lorentz reciprocal theorem for the homogeneous Stokes equations implies that $\mathbf{A}=\mathbf{A}^{T}, \mathbf{D}=\mathbf{D}^{T}$ and $\mathbf{B}^{T}=\mathbf{C}$ for any geometry. We refer to the right-handed orthonormal axes with the $y$ direction normal to the wall and the other directions parallel to the wall. Symmetry considerations imply that with respect to these axes $\mathbf{A}$ is diagonal, and $A_{22}=A_{33}$. The only non-zero elements of $\mathbf{B}$ are $B_{23}=-B_{32}$. The reciprocal theorem may be used to calculate $\mathbf{C}$ from $\mathbf{B}$. $\mathbf{D}$ is diagonal, and $D_{22}=D_{33}$ (Kim and Karrila, 2005). Then Eq. (5) is simplified as

$$
\left\{\begin{array}{l}
F_{1}=6 \pi \mu R A_{11} U_{1} \\
F_{2}=6 \pi \mu R\left(A_{22} U_{2}+R B_{23} \Omega_{3}\right) \\
F_{3}=6 \pi \mu R\left(A_{22} U_{3}-R B_{23} \Omega_{2}\right) \\
T_{1}=8 \pi \mu R^{2} R D_{11} \Omega_{1} \\
T_{2}=8 \pi \mu R^{2}\left(-B_{23} U_{3}+R D_{33} \Omega_{2}\right) \\
T_{3}=8 \pi \mu R^{2}\left(B_{23} U_{2}+R D_{33} \Omega_{3}\right)
\end{array}\right.
$$

Thus, the five left unknown coefficients are $A_{11}, A_{22}, B_{23}, D_{33}$ and $D_{11}$. The five coefficients of hydrodynamic force and torque at vanishing particle Reynolds number are listed in Table 2 (Dance and Maxey, 2003).

\subsection{New models for hydrodynamic force and torque at finite particle Reynolds numbers}

In this subsection, the new models for force and torque are proposed when a particle moves near a solid wall at finite particle Reynolds numbers whilst maintaining the form of formulae like Eq. (6). Due to the inherent nonlinearity of the governing equations of the flow at finite particle Reynolds number, Eq. (6) is no longer valid in that case. The unknown coefficients in Eq. (5) are 

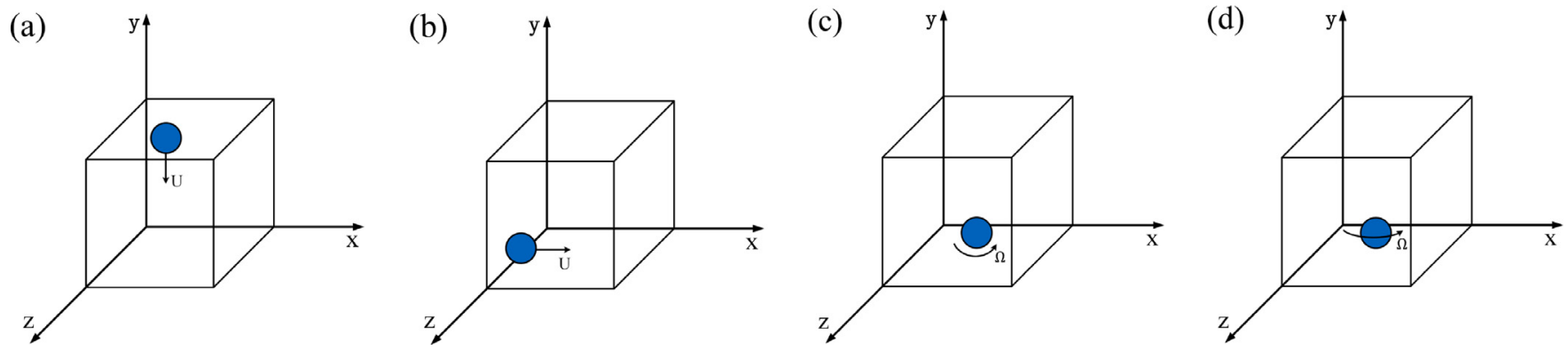

Fig. 1. A particle (a) moves along $y$ axis normal to the wall, (b) moves along $x$ axis parallel to the wall, (c) rotates around $z$ axis, (d) rotates around $y$ axis

much more complex. On one hand, the coefficients in Eq. (6) depend on both particle Reynolds number and the dimensionless gap. On the other hand, the coefficients denoting the lift force induced by the presence of the wall will not be zero any more and vary with particle Reynolds number and the dimensionless gap. Zeng et al. (2005) have extensively studied the dependence of the wallinduced hydrodynamic lift force using an accurate spectral element method and found that two different regimes of the dependence of the lift coefficient on particle Reynolds number. We will check and validate our numerical method by comparing the results on lift coefficient of Zeng et al. (2005) in Section 4.3. Here, we will focus on the dependence of leading coefficients of hydrodynamic force and torque on particle Reynolds number and the dimensionless gap in Eq. (6) using simple motions of a particle. Similar method has been used by researchers to propose drag models (Lin and Lin, 2013; Liu et al., 2009).

At finite particle Reynolds numbers, we could propose the following new models for the hydrodynamic force and torque on the particle,

$$
\left\{\begin{array}{l}
F_{1}=6 \pi \mu R A_{11}\left(R e_{p}, \varepsilon\right) U_{1} \\
F_{2}=6 \pi \mu R\left(A_{22}\left(R e_{p}, \varepsilon\right) U_{2}+R B_{23}\left(R e_{\Omega}, \varepsilon\right) \Omega_{3}\right) \\
F_{3}=6 \pi \mu R\left(A_{22}\left(R e_{p}, \varepsilon\right) U_{3}-R B_{23}\left(R e_{\Omega}, \varepsilon\right) \Omega_{2}\right) \\
T_{1}=8 \pi \mu R^{2}\left(R D_{11}\left(R e_{\Omega}, \varepsilon\right) \Omega_{1}\right) \\
T_{2}=8 \pi \mu R^{2}\left(-B_{23}\left(R e_{\Omega}, \varepsilon\right) U_{3}+R D_{33}\left(R e_{\Omega}, \varepsilon\right) \Omega_{2}\right) \\
T_{3}=8 \pi \mu R^{2}\left(B_{23}\left(R e_{\Omega}, \varepsilon\right) U_{2}+R D_{33}\left(R e_{\Omega}, \varepsilon\right) \Omega_{3}\right) .
\end{array}\right.
$$

In the new models, the coefficients $A_{11}, A_{22}, B_{23}, D_{11}, D_{33}$ are not only functions of the dimensionless gap $\varepsilon$, but also functions of the translational particle Reynolds number $R e_{p}$ or the rotational particle Reynolds number $R e_{\Omega}$. In the following subsection, we shall present the procedure to obtain the coefficients of the new models for hydrodynamic force and torque using given simple motions of a particle near a solid wall.

\subsection{Procedure to obtain the coefficients of the new models for hydrodynamic force and torque}

In the motion of a finite-size particle in an otherwise quiescent ambient fluid bounded by solid walls at the bottom and four lateral side walls, four simple types of particle motion near the solid wall at the bottom as shown in Fig. 1 are used to obtain the models for hydrodynamic force and torque at finite particle Reynolds number: (1) particle moving along $y$ axis normal to the bottom wall (Fig. 1(a)), (2) particle moving along $x$ axis parallel to the bottom wall (Fig. 1(b)), (3) particle rotating around $z$ axis parallel to the bottom wall (Fig. 1(c)), (4) particle rotating around $y$ axis normal to the bottom wall (Fig. 1(d)). The procedures of above mentioned four simple types of particle motion to be considered are illustrated in detail as follows:
2.3.1. Particle moving normal to the wall $\left(U_{1}=-U, U_{2}=0, \Omega_{3}=0\right)$

The particle starts to move along $y$ axis normal to the wall with a constant acceleration from a zero velocity, as depicted in Fig. 1(a). It moves at a constant speed after its velocity reaches a prescribed value. When the gap size between the particle and wall is small enough, we resolve the hydrodynamic force and calculate the coefficient $A_{11}$ as a function of $R e_{p}$ and $\varepsilon$

$F_{1}=6 \pi \mu R U_{1} \cdot A_{11}\left(R e_{p}, \varepsilon\right)$.

2.3.2. Particle moving parallel to the wall $\left(U_{1}=0, U_{2}=U, \Omega_{3}=0\right)$

The particle starts to accelerate along $x$ axis parallel to the wall with a constant acceleration from a zero velocity, as depicted in Fig. 1(b). It moves at a constant speed after its velocity reaches a prescribed value. We set different gaps between the particle and wall. The coefficient $A_{22}$ as a function of $\operatorname{Re}_{p}$ and $\varepsilon$ could be calculated with

$F_{2}=6 \pi \mu R U_{2} \cdot A_{22}\left(R e_{p}, \varepsilon\right)$.

\subsubsection{Particle rotating around an axis parallel to the wall}

$\left(U_{1}=0, U_{2}=0, \Omega_{3}=\Omega\right)$

The particle firstly rotates around $z$ axis with a constant angular acceleration from a zero angular velocity, as depicted in Fig. 1(c). It keeps rotating at a constant angular velocity after its angular velocity reaches a prescribed value. We set different gaps between the particle and wall. The coefficients $B_{23}$ and $D_{33}$ could be calculated with

$\left\{\begin{array}{l}F_{2}=6 \pi \mu R \cdot\left(R B_{23}\left(R e_{\Omega}, \varepsilon\right) \Omega_{3}\right) \\ T_{3}=8 \pi \mu R^{2} \cdot\left(R D_{33}\left(R e_{\Omega}, \varepsilon\right) \Omega_{3}\right) .\end{array}\right.$

\subsubsection{Particle rotating around an axis normal to the wall}

$\left(U_{1}=0, U_{2}=0, \Omega_{1}=\Omega\right)$

The particle starts to rotate around $y$ axis with a constant angular acceleration from a zero angular velocity, as depicted in Fig. 1(d). It keeps rotating at a constant angular velocity after its angular velocity reaches a prescribed value. We set different gaps between the particle and wall. The coefficient $D_{11}$ as a function of $R e_{\Omega}$ and $\varepsilon$ could be calculated with

$T_{1}=8 \pi \mu R^{2} \cdot\left(R D_{11}\left(\operatorname{Re}_{\Omega}, \varepsilon\right) \Omega_{1}\right)$.

\section{Numerical method}

The lattice Boltzmann method has been a popular numerical method for dynamical coupling between solid and fluid with complex geometrical boundaries (Rong et al., 2008; Zhu et al., 2011; Qi et al., 2014; Wu et al., 2014). In this work, the multiple-relaxationtime lattice Boltzmann method (MRT-LBM) is used to simulate the flow induced by a moving finite-size particle. The particle moves according to a prescribed acceleration or velocity as shown in Fig. 1, therefore, the coefficients from the force and torque on the particle can be calculated. Besides, different particle Reynolds 

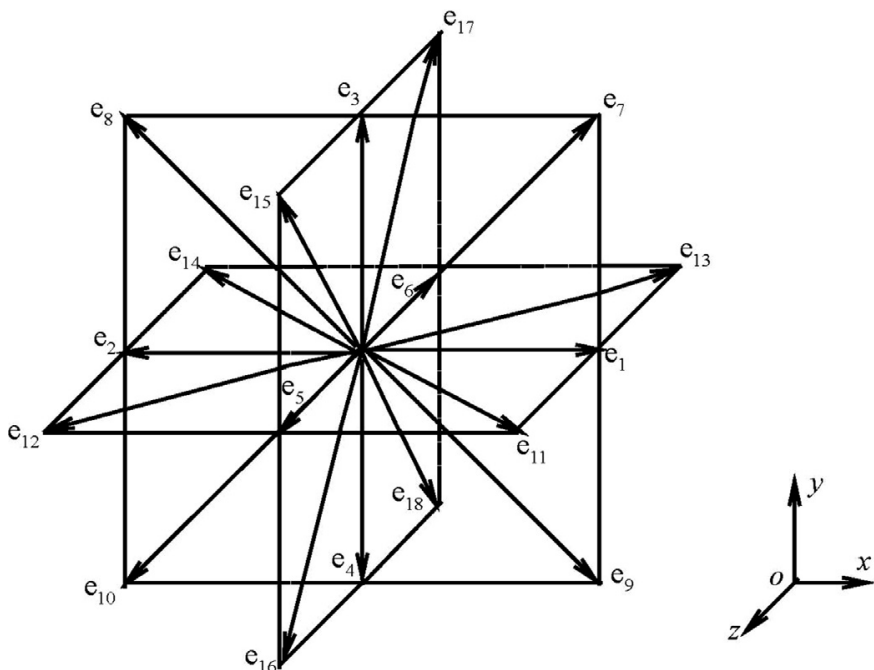

Fig. 2. Schematic diagram showing the velocities of 19 fluid lattice particles in the D3Q19 model.

numbers and gaps between the particle and wall in the parameter space are set $\left\{R e_{p}, R e_{\Omega}, \varepsilon\right\}$.

\subsection{Lattice Boltzmann method}

The MRT-LBM applies multiple relaxation times in collision process. At each lattice point $\mathbf{x}$ and time $t$, the mesoscale distribution function $\mathbf{f}(\mathbf{x}, t)$ is governed by (d'Humieres et al. (2002))

$\mathbf{f}\left(\mathbf{x}+\mathbf{e}_{i} \delta_{t}, t+\delta_{t}\right)=\mathbf{f}(\mathbf{x}, t)-\mathbf{M}^{-1} \cdot \mathbf{S} \cdot\left[\mathbf{m}-\mathbf{m}^{(e q)}\right]$,

where $\mathbf{f}(\mathbf{x}, t)$ is a vector indicating the distributions of lattice particle. In the D3Q19 discrete velocity model, $\mathbf{M}$ is a $19 \times 19$ orthogonal transformation matrix which converts the distribution function f from the discrete velocity space into the moment space $\mathbf{m}$, where the collision relaxation is performed. $\mathbf{m}^{(e q)}$ is the equilibrium value of the moment $\mathbf{m}$, and $\delta_{t}$ is the time step. The transformations between the particle velocity space and the moment space are

$\mathbf{m}=\mathbf{M} \cdot \mathbf{f}, \quad \mathbf{m}^{(e q)}=\mathbf{M} \cdot \mathbf{f}^{(e q)}, \quad \mathbf{f}=\mathbf{M}^{-1} \cdot \mathbf{m}$.

The macroscale variables are obtained from the moments of the distribution function $\mathbf{f}$ using

$\rho_{f 0}=1, \quad \rho_{f}=\sum_{i} f_{i}, \quad \rho_{f 0} \mathbf{u}=\sum_{i} f_{i} \mathbf{e}_{i}, \quad p=\rho_{f} c_{s}^{2}$,

where $\mathbf{u}$ is the macroscale fluid velocity, $\rho_{f 0}=1$ is the mean density, $p$ is the fluid pressure, $c_{s}=1 / \sqrt{3}$ is the speed of sound, $\mathbf{e}_{i}$ in the D3Q19 model shown in Fig. 2 can be expressed as

$\mathbf{e}_{i}= \begin{cases}(0,0,0) & i=1 \\ ( \pm 1,0,0),(0, \pm 1,0),(0,0, \pm 1) & i=1,2, \cdots, 6 \\ ( \pm 1, \pm 1,0),( \pm 1,0, \pm 1),(0, \pm 1, \pm 1) & i=7,8, \cdots, 18 .\end{cases}$

The elements of the transform matrix $\mathbf{M}$ can be found in d'Humieres et al. (2002). The 19 elements in $\mathbf{m}$ are

$$
\begin{aligned}
\mathbf{m}= & \left\{\rho_{f}, k_{1}, k_{2}^{2}, u_{x}, q_{x}, u_{y}, q_{y}, u_{z}, q_{z}, 3 p_{x x}, 3 \pi_{x x},\right. \\
& \left.p_{w w}, \pi_{w w}, p_{x y}, p_{y z}, p_{x z}, m_{x}, m_{y}, m_{z}\right\}^{T},
\end{aligned}
$$

where the element $m_{i}(i=0,1,2, \cdots, 18)$ respectively denote the fluid mass density $\rho_{f}$, the part of the kinetic energy $k_{1}$ independent of the density, the part of the kinetic energy square $k_{2}^{2}$ independent of the density and kinetic energy, the momentum $\rho_{f 0} u_{x}$, $\rho_{f 0} u_{y}, \rho_{f 0} u_{z}$, the energy flux $q_{x}, q_{y}, q_{z}$, the stress tensor $p_{x x}, p_{w w}$, $p_{x y}$ and third order moment $m_{x}, m_{y}, m_{z}$. The conserved hydrodynamic moments are the density and the momentum $m_{0}^{(e q)}=\rho_{f}$, $m_{3}^{(e q)}=\rho_{f 0} u_{x}, m_{5}^{(e q)}=\rho_{f 0} u_{y}$ and $m_{7}^{(e q)}=\rho_{f 0} u_{z}$, while other nonconserved kinetic moments are the functions of the conserved moments. The diagonal matrix $\mathbf{S}$ specifies the relaxation rates in collision for the non-conserved moments and,

$\mathbf{S} \equiv \operatorname{diag}\left(s_{0}, s_{1}, s_{2}, s_{3}, s_{4}, s_{3}, s_{4}, s_{3}, s_{4}, s_{9}, s_{10}, s_{9}, s_{10}\right.$,

$$
\left.s_{13}, s_{13}, s_{13}, s_{16}, s_{16}, s_{16}\right) \text {, }
$$

where $s_{0}=s_{3}=0$ for the conserved moments, and $s_{1}=1.19$, $s_{2}=s_{10}=1.4, \quad s_{4}=1.2, \quad s_{16}=1.98, \quad s_{9}=s_{13}=1 /(3 v+0.5)$ for the non-conserved moments, $v$ is the kinematic viscosity of the fluid (d'Humieres et al., 2002). With the speed of sound $c_{s}=1 / \sqrt{3}$ and $s_{9}=s_{13}$, the equilibrium values of the nonconserved moments are $m_{1}^{(e q)}=-11 \rho_{f}+19\left(u_{x}^{2}+u_{y}^{2}+u_{z}^{2}\right), m_{2}^{(e q)}=$ $w_{\varepsilon} \rho_{f}+w_{\varepsilon j}\left(u_{x}^{2}+u_{y}^{2}+u_{z}^{2}\right), \quad m_{4}^{(e q)}=-2 u_{x} / 3, \quad m_{6}^{(e q)}=-2 u_{y} / 3$, $m_{8}^{(e q)}=-2 u_{z} / 3, \quad m_{9}^{(e q)}=2 u_{x}^{2}-\left(u_{y}^{2}+u_{z}^{2}\right), \quad m_{10}^{(e q)}=w_{x x} m_{9}^{(e q)}$, $m_{11}^{(e q)}=u_{y}^{2}-u_{z}^{2}, \quad m_{12}^{(e q)}=w_{x x} m_{11}^{(e q)}, \quad m_{13}^{(e q)}=u_{x} u_{y}, \quad m_{14}^{(e q)}=u_{y} u_{z}$, $m_{15}^{(e q)}=u_{x} u_{z}, \quad m_{16}^{(e q)}=m_{17}^{(e q)}=m_{18}^{(e q)}=0$, where $w_{\varepsilon}, w_{\varepsilon j}$ and $w_{x x}$ are free parameters. They are set to $w_{\varepsilon}=0, w_{\varepsilon j}=-475 / 63$, and $w_{x x}=0$ for the optimized stability.

The following equilibrium distribution function is used to initialize the distribution function,

$f_{i}^{(e q)}=W_{i}\left(\rho_{f}+\rho_{f 0} \frac{\mathbf{e}_{i} \cdot \mathbf{u}}{c_{s}^{2}}+\rho_{f 0} \frac{\left(\mathbf{e}_{i} \cdot \mathbf{u}\right)^{2}}{2 c_{s}^{4}}-\rho_{f 0} \frac{u^{2}}{2 c_{s}^{2}}\right)$,

where the weight factor $W_{i}=1 / 3$ for $i=0 ; W_{i}=1 / 18$ for $i=$ $1, \cdots, 6$; and $W_{i}=1 / 36$ for $i=7, \cdots, 18$.

\subsection{Boundary condition}

For convenient implementation, the bottom wall and side walls of the container are located half lattice unit away from the lattice points. In this case, the no-slip boundary condition is simply implemented using the classic mid-point bounce-back scheme. Likewise, the top free surface is located at half lattice unit away from the lattice points and the free-slip boundary condition is implemented by the perfect reflective bounce-back. The no-slip boundary condition between the moving particle surface and fluid is implemented using the quadratic interpolation scheme (Bouzidi et al., 2001; Lallemand and Luo, 2003). When the particle is close to the wall and if there are only two lattice points in the gap between surfaces, the linear interpolation scheme is used Bouzidi et al. (2001). The interpolated bounce-back scheme produces a relatively smooth sphere surface instead of a staircase shaped surface when the mid-point bounce back scheme is used on the particle surface (Ladd, 1994a; 1994b). It can also reduce the nonphysical fluctuation of the sphere surface due to the motion of the sphere relative to the fixed lattice grid points. When the gap becomes even smaller and there is only one lattice point in it, the simple bounce back scheme is used to calculate the unknown distribution functions.

In the simulation as documented in this work, the fluid lattice points are fixed in space while the solid spherical particle moves relative to the fixed lattice grids. A lattice node previously located inside the solid particle may become a fluid node due to the motion of the solid particle. The distribution functions for such new fluid nodes have to be constructed. In our simulation, all the 19 components of the distribution function for a new fluid node are constructed with an equilibrium distribution plus a nonequilibrium correction (Caiazzo, 2008). The equilibrium distribution part is calculated using Eq. (18) based on the velocity of the moving boundary of a particle $\mathbf{u}_{w}$ and the fluid density, $\bar{\rho}_{f}$, averaged over all the existing fluid nodes in the immediate neighborhoods. The non-equilibrium part is obtained from a neighboring 
fluid node along the direction of a discrete velocity $\mathbf{e}_{i}$ which maximizes the quantity $\mathbf{e}_{i} \cdot \hat{\mathbf{n}}$, where $\hat{\mathbf{n}}$ is the local outward-normal vector of the moving boundary at the point through which the lattice node moves out to the fluid region (Caiazzo, 2008). Further details are given in Gao et al. (2013).

\subsection{Hydrodynamic force and torque}

In this research work, the motion of particle with a prescribed velocity or angular velocity is being controlled. Then the hydrodynamic force and torque acting on the particle can be calculated using the theorem of impulse. In the conventional CFD methods, the hydrodynamic force is calculated by integrating the local stress over the particle surface which is calculated by spatial differentiations of the fluid velocity. An advantage of LBM is that the hydrodynamic force and torque acting on the solid particle is directly calculated based on the impulses exerted on the lattice fluid particles and the Newton's third law. The hydrodynamic force $\mathbf{F}_{h y}$ acting on the solid particle is the summation of the loss of the fluid momentum, from $t$ to $t+\delta_{t}$, on all the links cutting the solid particle surface. The torque $\mathbf{T}_{h y}$ is the summation of the cross product of the local position vector relative to the center of the particle and the loss of fluid momentum over all boundary links. Namely,

$$
\begin{aligned}
\mathbf{F}_{h y}^{t+0.5 \delta_{t} \delta_{t}} & =\sum_{\text {bn }}\left[\hat{f}_{i}(\mathbf{x}, t) \mathbf{e}_{i}-f_{\bar{i}}\left(\mathbf{x}, t+\delta_{t}\right) \mathbf{e}_{\bar{i}}\right] \\
& =\sum_{\text {bn }}\left[\hat{f}_{i}(\mathbf{x}, t)+f_{\bar{i}}\left(\mathbf{x}, t+\delta_{t}\right)\right] \mathbf{e}_{i}, \\
\mathbf{T}_{h y}^{t+0.5 \delta_{t}} \delta_{t} & =\sum_{\text {bn }} \mathbf{n}_{i} \times\left[\hat{f}_{i}(\mathbf{x}, t)+f_{\bar{i}}\left(\mathbf{x}, t+\delta_{t}\right)\right] \mathbf{e}_{i},
\end{aligned}
$$

where "bn" denotes summation over all the boundary links, $\mathbf{e}_{\bar{i}}=$ $-\mathbf{e}_{i}$ and $\hat{\mathbf{f}}(\mathbf{x}, t)=\mathbf{f}(\mathbf{x}, t)-\mathbf{M}^{-1} \cdot \mathbf{S} \cdot\left[\mathbf{m}-\mathbf{m}^{(e q)}\right]$ denotes the distribution function just after the collision (referred to the time step $\left.t+0.5 \delta_{t}\right), f_{\bar{i}}\left(\mathbf{x}, t+\delta_{t}\right)$ denotes the distribution function after bounce-back collision with the solid particle surface at $x$ and $t+\delta_{t}$, $\hat{\mathbf{n}}_{i}$ denotes the local outward-normal vector connecting the solid particle center and the point of intersection between the $i$ link at $x$ with the surface of the solid particle.

After obtaining the hydrodynamic force and torque acting to a particle, we update the particle transversal velocity $\mathbf{V}_{p}^{t+\delta_{t}}$, particle rotational velocity $\boldsymbol{\Omega}_{p}^{t+\delta_{t}}$, particle transversal displacement $\mathbf{Y}_{p}^{t+\delta_{t}}$ and particle angular displacement $\boldsymbol{\Theta}_{p}^{t+\delta_{t}}$, respectively, by

$\mathbf{V}_{p}^{t+\delta_{t}}=\mathbf{V}_{p}^{t}+\frac{1}{2 M_{p}}\left[\mathbf{F}_{h y}^{t+\delta t / 2}+\mathbf{F}_{h y}^{t-\delta_{t} / 2}\right] \delta_{t}+\mathbf{g}\left(1-\frac{\rho_{f 0}}{\rho_{p}}\right) \delta_{t}$,

$\boldsymbol{\Omega}_{p}^{t+\delta_{t}}=\boldsymbol{\Omega}_{p}^{t}+\frac{1}{2 I_{p}}\left[\boldsymbol{\Gamma}_{h y}^{t+\delta t / 2}+\boldsymbol{\Gamma}_{h y}^{t-\delta_{t} / 2}\right] \delta_{t}$,

$\mathbf{Y}_{p}^{t+\delta_{t}}=\mathbf{Y}_{p}^{t}+0.5\left(\mathbf{V}_{p}^{t}+\mathbf{V}_{p}^{t+\delta_{t}}\right) \delta_{t}$

$\boldsymbol{\Theta}_{p}^{t+\delta_{t}}=\boldsymbol{\Theta}_{p}^{t}+0.5\left(\boldsymbol{\Omega}_{p}^{t}+\boldsymbol{\Omega}_{p}^{t+\delta_{t}}\right) \delta_{t}$,

where $M_{p}$ denotes the mass of the particle and $I_{p}$ the moment of inertia of the particle, $\mathbf{g}$ the gravitational acceleration, $\rho_{f 0}$ and $\rho_{p}$ fluid and particle density, respectively. For the four simple types of particle motion in this paper, $\mathbf{V}_{p}^{t}, \mathbf{V}_{p}^{t+\delta_{t}}=\mathbf{V}_{p}^{t}+\mathbf{a}_{p, t} \delta_{t}, \boldsymbol{\Omega}_{p}^{t}, \boldsymbol{\Omega}_{p}^{t+\delta_{t}}=$ $\boldsymbol{\Omega}_{p}^{t}+\mathbf{a}_{p, r} \delta_{t}$ are respectively the translational velocity and rotational velocity of the particle at time $t$ and $t+\delta_{t}$, and $\mathbf{a}_{p, t}$ and $\mathbf{a}_{p, r}$ are the prescribed translational acceleration and rotational acceleration of particle to be accelerated to a given velocity from a zero ve- locity for the purpose of numerical stability. When the particle is accelerated to a prescribed value, it will translate or rotate with a constant velocity, $\mathbf{V}_{p}^{t+\delta_{t}}=\mathbf{V}_{p}^{t}, \boldsymbol{\Omega}_{p}^{t+\delta_{t}}=\boldsymbol{\Omega}_{p}^{t}$ to obtain a statistically steady hydrodynamic force and torque.

\subsection{Transformation between physical unit and lattice Boltzmann unit}

To guarantee the numerical stability, we estimate the maximum value of fluid velocity to limit the Mach number of the flow such that $\max \left\{u_{B}\right\} / c_{S} \leq 0.15$, where $u_{B}$ is the prescribed velocity of particle in lattice Boltzmann unit. The value of $u_{B}$ is set based on the balance between forces in steady settling process and obtain different particle Reynolds numbers by varying the kinematic viscosity in lattice Boltzmann unit.

The formula of drag force on the particle in Stokes flow is $F=-6 \pi \mu R u$. When the particle Reynolds number becomes large enough, the nonlinear drag formula becomes $F=-6 \pi \mu R u f\left(\operatorname{Re}_{p}\right)$, where $f\left(R e_{p}\right)=1+0.15 R e_{p}^{0.687}$ (Clift et al., 1978), $u$ is the prescribed velocity of a particle. The drag force, gravity and buoyancy on the particle under the equilibrium condition satisfy:

$6 \pi \mu R u f\left(\operatorname{Re}_{p}\right)=\frac{4}{3} \pi R^{3}\left(\rho_{p}-\rho_{f}\right) g$,

where $\rho_{p}=1500 \mathrm{~kg} / \mathrm{m}^{3}$ is the density of particle.

The velocity $u$ in Eq. (25) can be solved using Newtonian iteration method and the particle Reynolds number will be computed. According to the dimensional analysis, the transformation of quantities between physical unit and lattice Boltzmann unit is depicted as

$\frac{T_{p}}{T_{B}}=\frac{d_{p}}{d_{B}} \cdot \frac{u_{B}}{u_{p}}, \quad \frac{v_{p}}{v_{B}}=\frac{d_{p}}{d_{B}} \cdot \frac{u_{p}}{u_{B}}$.

In Eq. (26), the subscript " $p$ " and " $B$ " represent the physical and lattice Boltzmann unit respectively: $d_{p}=6 \mathrm{~mm}, v_{p}=\mu / \rho_{f}, u_{p}$ is the velocity in Eq. (25), $u_{B}$ and $d_{B}=2 r_{B}=50$ are respectively the prescribed velocity and diameter of the particle in lattice Boltzmann unit. Besides, the rotational velocity of the particle is $\Omega=$ $u / R$, where $u$ is the maximum linear velocity at the surface of a rotating particle. Therefore we can obtain all physical parameters corresponding to different particle Reynolds numbers, as shown in Table 3.

When the location, velocity, force and torque on a particle are obtained, we then apply Eqs. (8)-(11) in Section 2 to compute the coefficients of hydrodynamic force and torque.

\section{Results and discussions}

\subsection{Computational domain size}

Before proposing new models for hydrodynamic force and torque at finite particle Reynolds numbers, a compromise between the computational domain size and the needed computational cost have to be made. It's necessary to guarantee that the domain size has little influence on the results. Therefore, the results are checked by varying the computational domain size and fixing all other parameters. We make comparisons of the coefficients of hydrodynamic force and torque for different computational domain sizes, depicted as Figs. 3 and 4. The three-dimensional sizes of computational domain are respectively denoted as $L_{x}, L_{y}$ and $L_{z}$ in lattice Boltzmann unit.

In Fig. 3, the different lines denote the coefficient $A_{11}$ versus the dimensionless gap $\varepsilon$ under the condition of computational domain sizes $L_{X} / d_{B}=L_{z} / d_{B}=6.6,8,9$, and 10 for the particle moving along $y$ axis normal to the wall at $R e_{p}=140.719$. $d_{B}=50$ denotes the resolution of particle diameter in lattice Boltzmann unit. In this 
Table 3

The particle Reynolds numbers and the corresponding parameters in physical unit and lattice Boltzmann unit.

\begin{tabular}{|c|c|c|c|c|c|c|c|c|}
\hline Case & $\rho_{f}\left(\mathrm{~kg} / \mathrm{m}^{3}\right)$ & $\mu\left(10^{-3} \mathrm{~N} \cdot \mathrm{s} / \mathrm{m}^{2}\right)$ & $v_{B}$ & $u_{p}(\mathrm{~m} / \mathrm{s})$ & $r_{B}$ & $u_{B}$ & St & $R e_{p}$ \\
\hline Case 1 & 967 & 290 & 0.38551480 & 0.032413 & 25 & 0.005 & 0.112 & 0.6485 \\
\hline Case 2 & 965 & 212 & 0.21577852 & 0.042422 & 25 & 0.005 & 0.200 & 1.159 \\
\hline Case 3 & 962 & 113 & 0.14221879 & 0.068828 & 25 & 0.01 & 0.609 & 3.516 \\
\hline Case 4 & 960 & 58 & 0.09645247 & 0.104398 & 25 & 0.02 & 1.8 & 10.368 \\
\hline Case 5 & 950 & 28 & 0.03278679 & 0.149825 & 25 & 0.02 & 5.351 & 30.500 \\
\hline Case 6 & 930 & 15 & 0.02077498 & 0.194092 & 25 & 0.03 & 12.939 & 72.202 \\
\hline Case 7 & 925 & 9 & 0.01421272 & 0.228193 & 25 & 0.04 & 25.355 & 140.719 \\
\hline Case 8 & 915 & 6 & 0.00846908 & 0.258091 & 25 & 0.04 & 43.015 & 236.153 \\
\hline Case 9 & 910 & 5 & 0.00841330 & 0.272114 & 25 & 0.05 & 54.423 & 297.148 \\
\hline Case 10 & 900 & 4.2 & 0.00675197 & 0.287982 & 25 & 0.05 & 68.567 & 370.263 \\
\hline
\end{tabular}

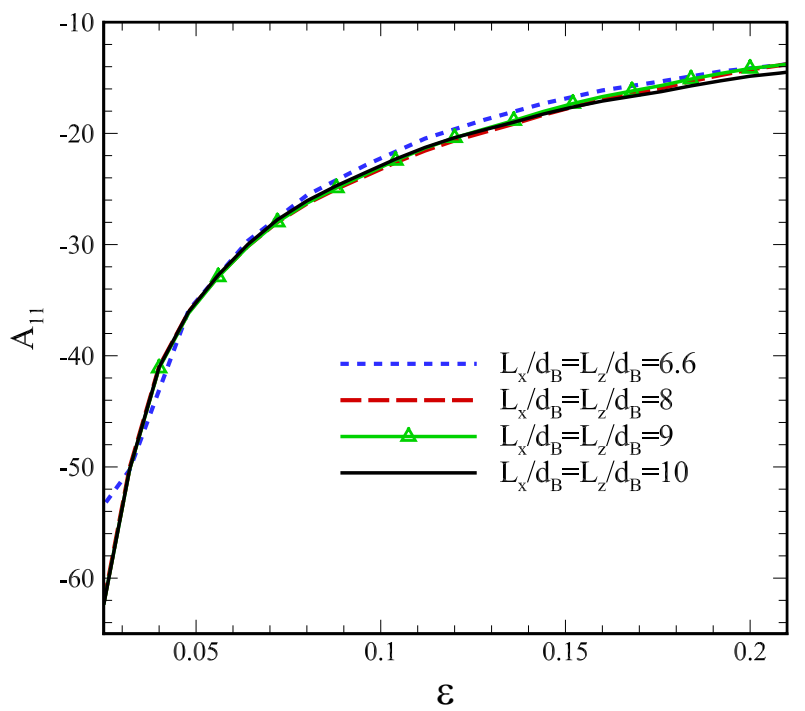

Fig. 3. Effects of computational domain size on the coefficient $A_{11}$ of drag force as a function of dimensionless gap $\varepsilon$.

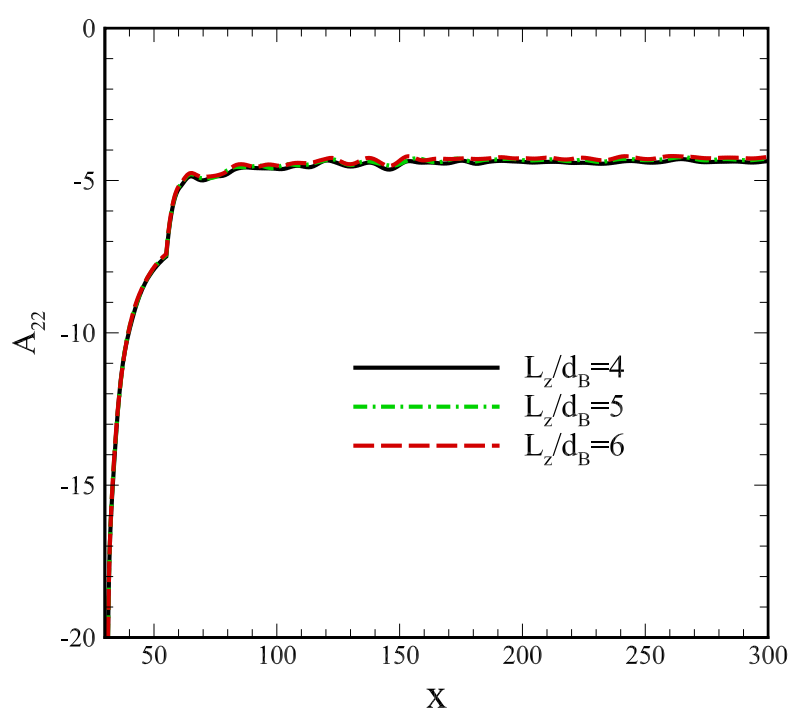

Fig. 4. Effects of computational domain size on the coefficient $A_{22}$ of drag force as a function of $x$-coordinate of the particle for $\varepsilon=0.03$, where $x=30$ is the initial position of the particle.

type of particle motion, $y$-direction is the direction of particle motion and the value of $L_{y}$ is determined by the prescribed particle velocity and number of time steps. The symbol $\varepsilon=n_{g} / r_{B}$ denotes the dimensionless gap scaled by the radius of particle, $n_{g}$ and $r_{B}$ are the resolution of gap and particle radius in lattice Boltzmann unit. There is only a negligible difference between the curves of $L_{x} / d_{B}=L_{z} / d_{B}=8,9$ and 10 , so that $L_{x} / d_{B}=L_{z} / d_{B}=8$ is a feasible size for the type 1 .

Fig. 4 shows the curves of $A_{22}$ of a particle moving along $x$ axis in the flow domain at $\operatorname{Re}_{p}=30.500$ in type 2. The different lines denote the curves of $L_{z} / d_{B}=4,5$ and 6 with $L_{y} / d_{B}=4$. The gap size between the particle and the wall is 0.75 . At the beginning of the curves, the value of $A_{22}$ rapidly increases because the particle moves along $x$ axis with a constant acceleration. Then the particle moves at a constant velocity after reaching a prescribed value and the coefficient $A_{22}$ tends to be steady. The curves with $x$-coordinate from 150 to 300 are steady, where the equilibrium value of $A_{22}$ under stationary state could be obtained. From Fig. 4, the value of $L_{z} / d_{B}$ can be set to 4 .

After making comparisons, we can obtain optimal 3D sizes of computational domain for all types of particle motions. For the type 3 , we set $L_{x} / d_{B}=L_{z} / d_{B}=6, L_{y} / d_{B}=4$. For the type 4 , we set $L_{x} / d_{B}=L_{z} / d_{B}=4, L_{y} / d_{B}=3$.

\subsection{Grid-size convergence}

In order to obtain a grid-size independent result, the grid-size convergence is studied. For the particle of $d_{p}=2 R=6 \mathrm{~mm}$ rotating around $y$ and $z$ axes in fluid flow at $R e_{\Omega}=140.719$, a dimensionless gap $\varepsilon=h / R=0.04$ is fixed, which means the gap in physical unit is $h=0.12 \mathrm{~mm}$. In the simulations, the grid resolutions of particle radius are $r_{B}=d_{B} / 2=20,25,35,50$, corresponding to the grid resolution for the gap between the particle and the wall $n_{g}=0.8,1.0,1.4,2.0$ respectively. In Figs. 5 and 6 , the coefficients $B_{23}, D_{33}$ and $D_{11}$ as functions of $d_{p} / \Delta x$ and $n_{g}$ are shown, where $d_{p} / \Delta x$ is used as the lower horizontal axis, $n_{g}$ is used as the upper horizontal axis, and $\Delta x$ denotes the grid size. The particle resolution of $d_{p} / \Delta x=d_{B}=50$ can correctly compute the coefficients $D_{33}$ and $D_{11}$ with the errors of $1.8 \%$ and $0.9 \%$ respectively comparing to $d_{p} / \Delta x=100$. For the coefficient $B_{23}$, the small errors between different resolutions is also negligible. Therefore the grid resolution of $d_{p} / \Delta x=50$ is used to resolve the fluid flow and obtain the hydrodynamic force and torque.

\subsection{Coefficients of drag and lift forces induced by a wall}

Zeng et al. (2005) have extensively studied the lift force induced by a wall by performing particle-resolved direct numerical simulations of a rigid sphere translating parallel to a flat wall in an otherwise quiescent ambient fluid, which is the same as the second type of particle motion in this paper. In order to validate the accuracy of the current simulation method and results, we shall compare our simulation results with the data of Zeng et al. (2005). The grid resolution of particle radius is set to $r_{B}=15$ and the computational domain is given by $600 \times 210 \times 300$. The drag and lift 
(a)

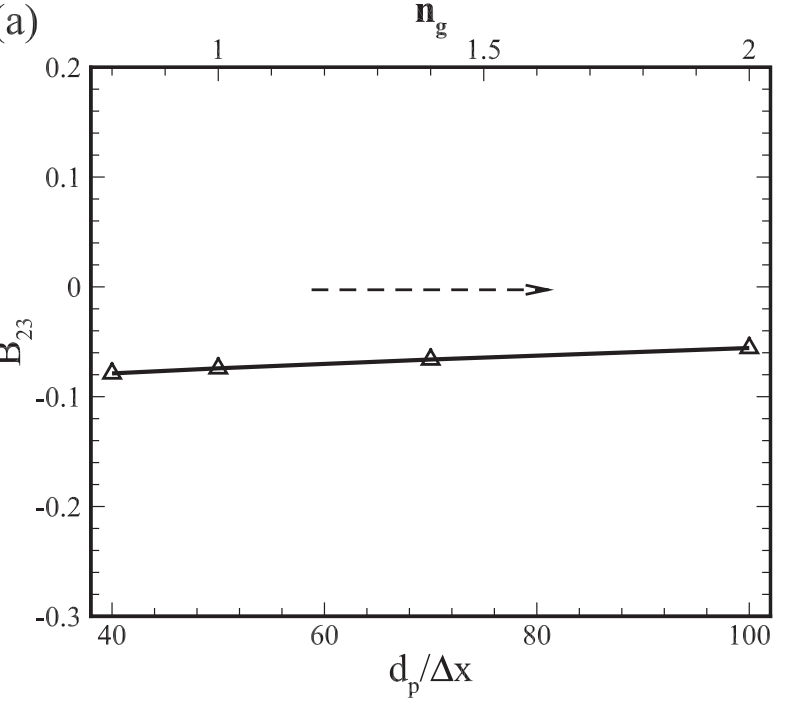

(b)

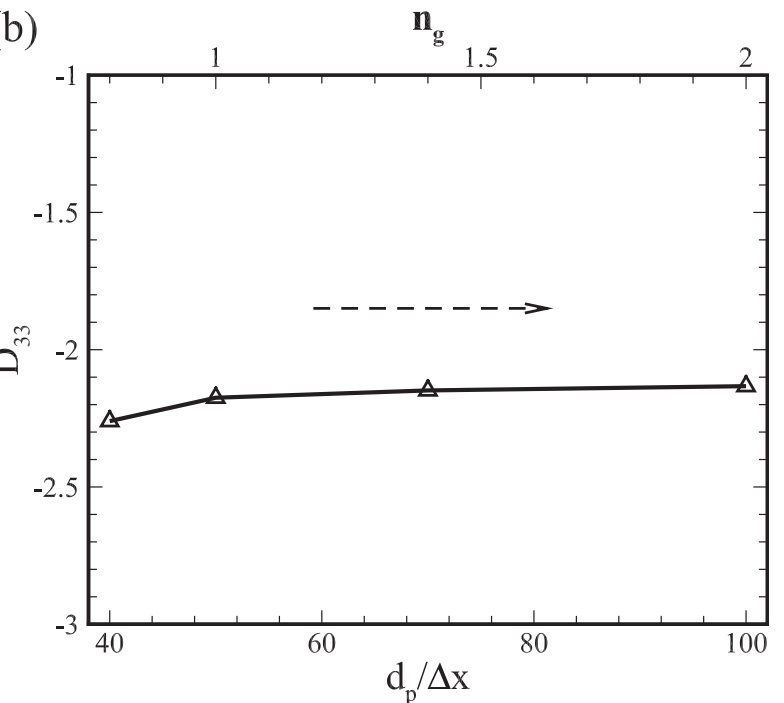

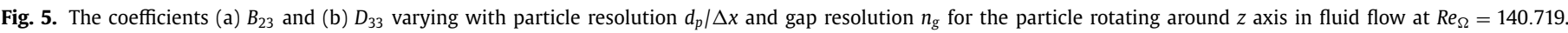

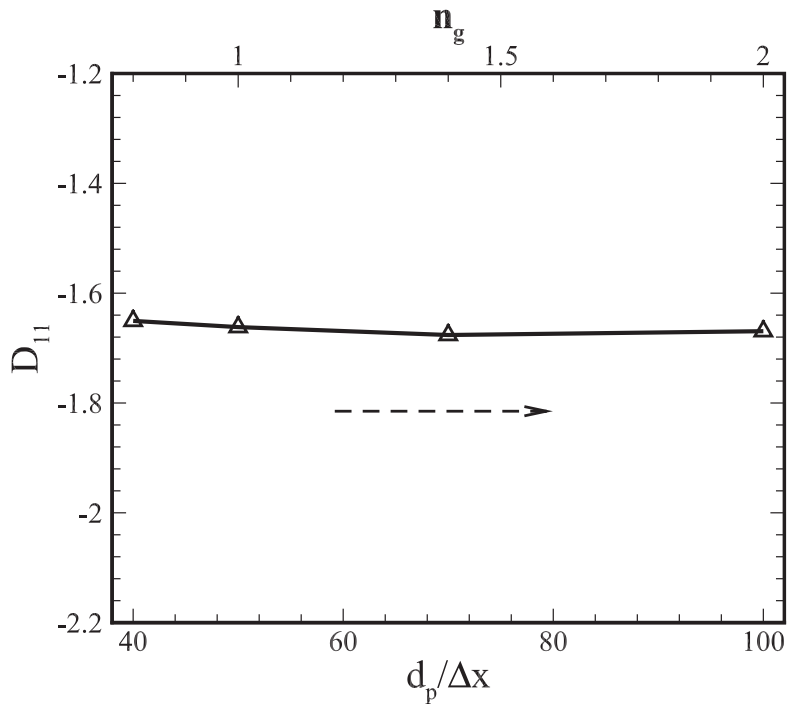

Fig. 6. The coefficient $D_{11}$ varying with particle resolution $d_{p} / \Delta x$ and gap resolution $n_{g}$ for the particle rotating around $y$ axis in fluid flow at $\operatorname{Re}_{\Omega}=140.719$.

coefficients are defined as follows:

$\left\{\begin{array}{l}C_{D}=\frac{F_{D}}{\frac{1}{2} \rho U^{2} \pi R^{2}}=A_{22} \cdot \frac{24}{R e_{p}} \\ C_{L}=\frac{F_{L}}{\frac{1}{2} \rho U^{2} \pi R^{2}},\end{array}\right.$,

where $F_{D}$ and $F_{L}$ are the drag force and lift force on the particle, respectively. Eq. (28) defines rescaled distance from the particle to the wall, $L^{*}$, by the particle Reynolds number.

$L=\frac{s+r_{B}}{2 r_{B}}=\frac{\varepsilon+1}{2}, \quad L^{*}=L \cdot R e_{p}$

Fig. 7 shows the drag and lift coefficients obtained from the present simulations and the results of Zeng et al. (2005). The solid line in Fig. 7(a) represents the standard drag correlation $C_{D}=$ $\frac{24}{\operatorname{Re}_{p}}\left(1+0.15 \operatorname{Re}_{p}^{0.687}\right)$ (Clift et al., 1978). Obviously, for all separations $(L=0.75,1,2)$ good agreements can be observed for different particle Reynolds numbers and different $L^{*}$ between the present simulations and the results of Zeng et al. (2005). The collapses between the current simulations and the results obtained from the spectral element method validate the accuracy of current lattice Boltzmann method.

\subsection{Gravity-driven settling of a particle}

In order to directly evaluate the particle motion and the fluid flow induced by the particle, we simulate a gravity-driven particle settling process and compare the simulation results with the experiments carried out by ten Cate et al. (2002). In the experiment, a small Nylon sphere of density $\rho_{p}=1120 \mathrm{~kg} / \mathrm{m}^{3}$ and diameter $d_{p}=15 \times 10^{-3} \mathrm{~m}$ was released in a tank filled with silicon oil at initial gap of $h_{0}=0.12 \mathrm{~m}$ from the bottom of the tank to the lowest point on the particle surface. The dimensions of the tank are $L_{x} \times L_{y} \times L_{z}=0.1 \mathrm{~m} \times 0.16 \mathrm{~m} \times 0.1 \mathrm{~m}$. We specifically simulate Case E1 and Case E4 for comparison. The experimental parameters are listed in Table 4, where the terminal velocity of a particle in an infinite medium $v_{p, t}$ is determined by a relation for the drag coefficient, $C_{d}=24\left(9.06 / \sqrt{R e_{p}}+1\right)^{2} / 9.06^{2}$ with $R e_{p}=d_{p} v_{p, t} / \nu$. In our simulation, 15 lattice grids are used to resolve the particle diameter.

Fig. 8 shows the vertical velocities $V_{p, y}$ of the particle from the moment of release for Case E1 and Case E4. The numerical results are in excellent agreement with the experimental data. We observe four different phases in the settling process: (a) the acceleration phase from rest due to the gravity, (b) the steady falling phase when the hydrodynamic force balances the effective gravity force (gravity minus the buoyancy force), (c) the deceleration phase due to the hydrodynamic pressure when the gap between the particle and the bottom wall becomes small, and (d) the final phase as the solid particle gradually loses its momentum at the bottom of the tank due to the viscous energy dissipation in the fluid.

The time history of the fluid velocity at a fixed location is compared with the experimental data in Figs. 9 and 10. The monitor point is positioned at one diameter from the bottom and one diameter from the vertical center line of the container in $x$ direction. The squeezing action of the particle pushes the liquid at the monitor point to move outwards with a positive peak (Fig. 9). After the vortex induced by the particle passes the monitor point, the fluid velocity at this point decreases. The fluid comes to rest very quickly after the particle comes to rest in Case E1, while in Case E4 a much larger wake forms due to the higher Reynolds number and the velocity slowly decays after the particle touches the bottom. 

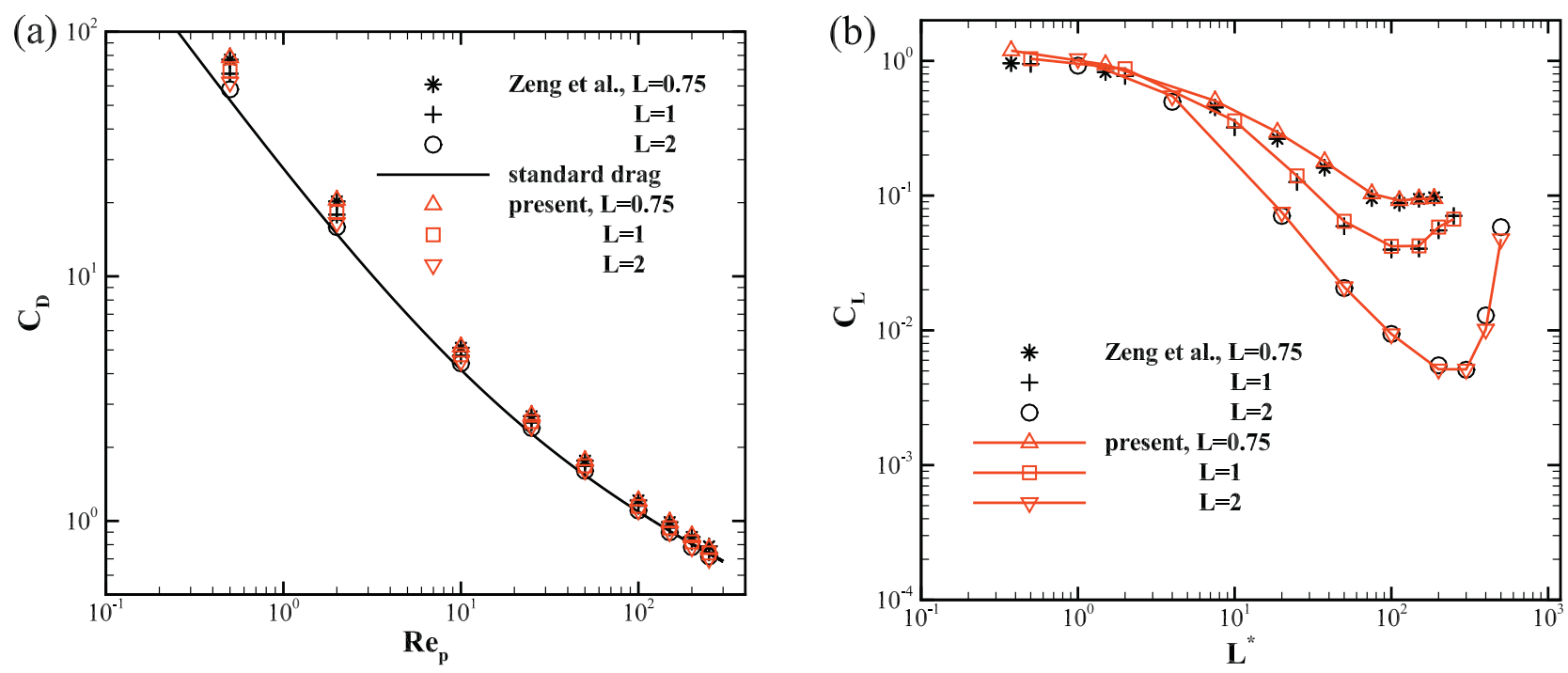

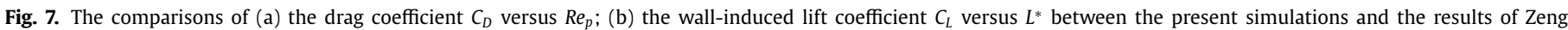
et al. for the scaled separation distance $L=0.75,1,2$.

Table 4

Parameters used in the simulated cases.

\begin{tabular}{llllllll}
\hline Case & $\rho_{f}\left(\mathrm{~kg} / \mathrm{m}^{3}\right)$ & $\mu\left(10^{-3} \mathrm{~N} \cdot \mathrm{s} / \mathrm{m}^{2}\right)$ & $\rho_{p}\left(\mathrm{~kg} / \mathrm{m}^{3}\right)$ & $d_{p}\left(10^{-3} \mathrm{~m}\right)$ & $v_{p, t}(\mathrm{~m} / \mathrm{s})$ & $R_{p}$ & $S t$ \\
\hline E1 & 970 & 373 & 1120.0 & 15 & 0.038 & 1.5 & 0.19 \\
E4 & 960 & 58 & 1120.0 & 15 & 0.128 & 31.9 & 4.13 \\
\hline
\end{tabular}

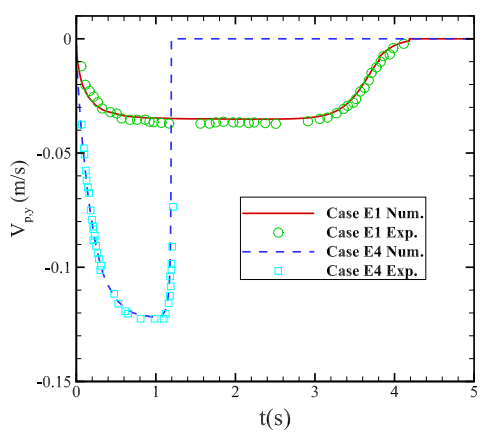

Fig. 8. The particle vertical velocity as a function of time. Lines are for the present simulation and symbols for the experimental measurement by ten Cate et al.

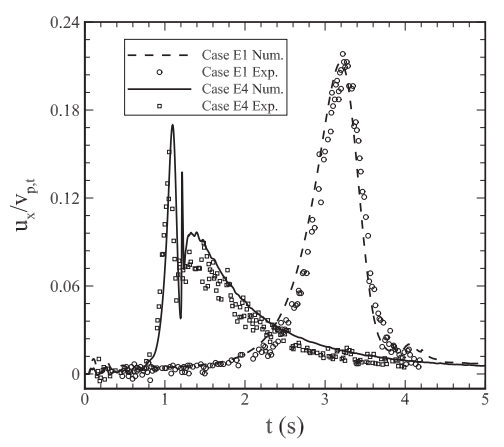

Fig. 9. Lateral fluid velocity induced by the particle at a monitor point as a function of time. Lines are for the present simulation and symbols for the experimental measurement by ten Cate et al.

Our simulations also well capture the vertical component of the fluid velocity due to downward motion of the particle (Fig. 10). Depending on the position of the vortex center relative to the monitor point and the particle center, the vertical velocity has different

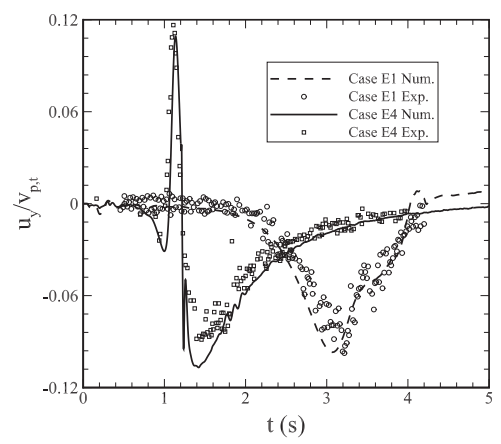

Fig. 10. Vertical fluid velocity induced by the particle at a monitor point as a function of time. Lines are for the present simulation and symbols for the experimental measurement by ten Cate et al.

transient behaviors. In Case E1, the Reynolds number is low and the region of the momentum diffusion in lateral direction is large during particle settling, the monitor point lies between the particle and the vortex center, thus the vertical velocity does not change sign. In Case E4, the Reynolds number is high and the region of momentum diffusion in lateral direction is small during particle settling, the monitor point lies on the right of vortex center, thus the vertical velocity changes its sign when the vortex center passes the monitor point (ten Cate et al., 2002).

The good agreements between the simulation results on particle motion and the fluid flow induced by the falling particle and the experimental data further validate the accuracy of the simulation method used in this study.

\subsection{Models for the hydrodynamic force and torque}

\subsubsection{Particle moving normal to the wall}

Firstly examined is the case that a spherical particle moves normal to the wall in the quiescent fluid. Figs. 11 and 12 

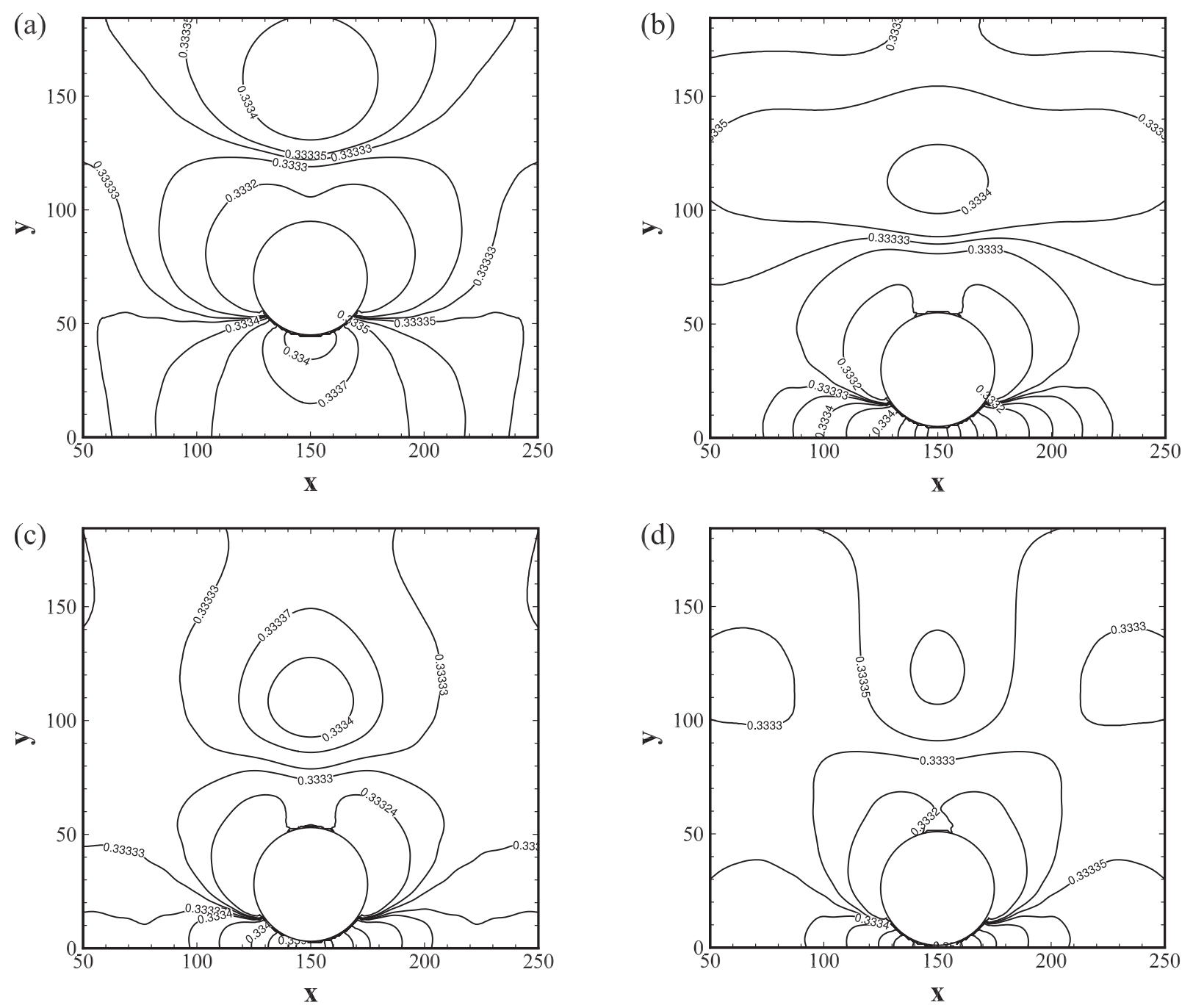

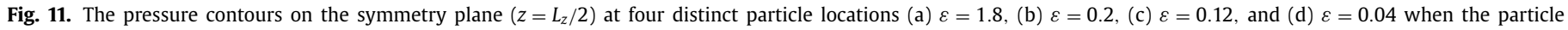
moves normal to the wall in fluid flow at $R e_{p}=140.719$.

plot the pressure contours and velocity vectors on the symmetry plane $\left(z=L_{z} / 2\right)$ for four distinct particle locations at $\varepsilon=$ $1.8,0.2,0.12,0.04$ when the particle moves in fluid flow at $\operatorname{Re}_{p}=$ 140.719. Fig. 11 shows that the highest and lowest pressures occurred at the front and the rear stagnation point of the sphere, respectively. In Fig. 11(a), the pressure contour is similar to that developed around the sphere in uniform flow without any solid boundary. When the particle moves closer to the wall, the pressure gradient in the interstitial liquid between the particle surface and the wall increases correspondingly to drive the flows. The high pressure gradient in the small gap increases the deformation of the pressure contours and the streamlines in Figs. 11(b)-(d) and 12(b)(d).

Furthermore, the relative velocity vector field $\left(u_{f}-U, w\right)$ is used to generate the streamlines that would be observed along a reference frame moving with the sphere when $\varepsilon=1.8$ as shown in Fig. 13. For different dimensionless gap $\varepsilon$ and $R e_{p}$, the numerical values of coefficient $A_{11}^{N}$ are calculated using Eq. (8), shown in Fig. 14(a) with discrete symbols. The models for $A_{11}$ expressed with $\varepsilon$ and $R e_{p}$ through data fitting could be obtained, as shown in Eq. (29). Eq. (30) is simply derived from Eq. (29). Here and hereafter, the superscript " $T$ " denotes theoretical results listed in Table 2.
$A_{11}=A_{11}^{T}+\left(6.07 \times 10^{-4}+0.0351 \cdot \ln \varepsilon\right) \cdot R e_{p}, \quad R e_{p} \leq 370$,

$A_{11}^{*}=\frac{A_{11}-A_{11}^{T}}{6.07 \times 10^{-4}+0.0351 \cdot \ln \varepsilon}=R e_{p}$

In Fig. 14(a), Eq. (29) is plotted with the curves of $A_{11}$ as a function of $\varepsilon$ at different particle Reynolds numbers. For each curve, the absolute value of coefficient $A_{11}$ increases with decreasing $\varepsilon$. When $R e_{p}$ is close to zero, the corrected formula of $A_{11}$ is consistent with the theoretical expression listed in line 2 of Table 2. The particle Reynolds number has a significant impact on the coefficient $A_{11}$. For a given $\varepsilon$, the absolute value of $A_{11}$ increases with $R e_{p}$ monotonically. Fig. 14(b) shows the normalized coefficient $A_{11}^{*}$ using the dimensionless gap as a function of $R e_{p}$ according to Eq. (30). The results of $A_{11}^{*}$ versus $R e_{p}$ collapse into a line with a slope of 1 , demonstrating the accuracy of data fitting.

Lin and Lin (2013) have numerically studied the effects of finite particle Reynolds numbers up to $R e_{p}=50$ on the drag coefficient on a particle moving towards a solid wall using the immersed boundary method. They used the Stokes force $-6 \pi \mu R U$ on the particle to obtain drag coefficient and proposed the following 

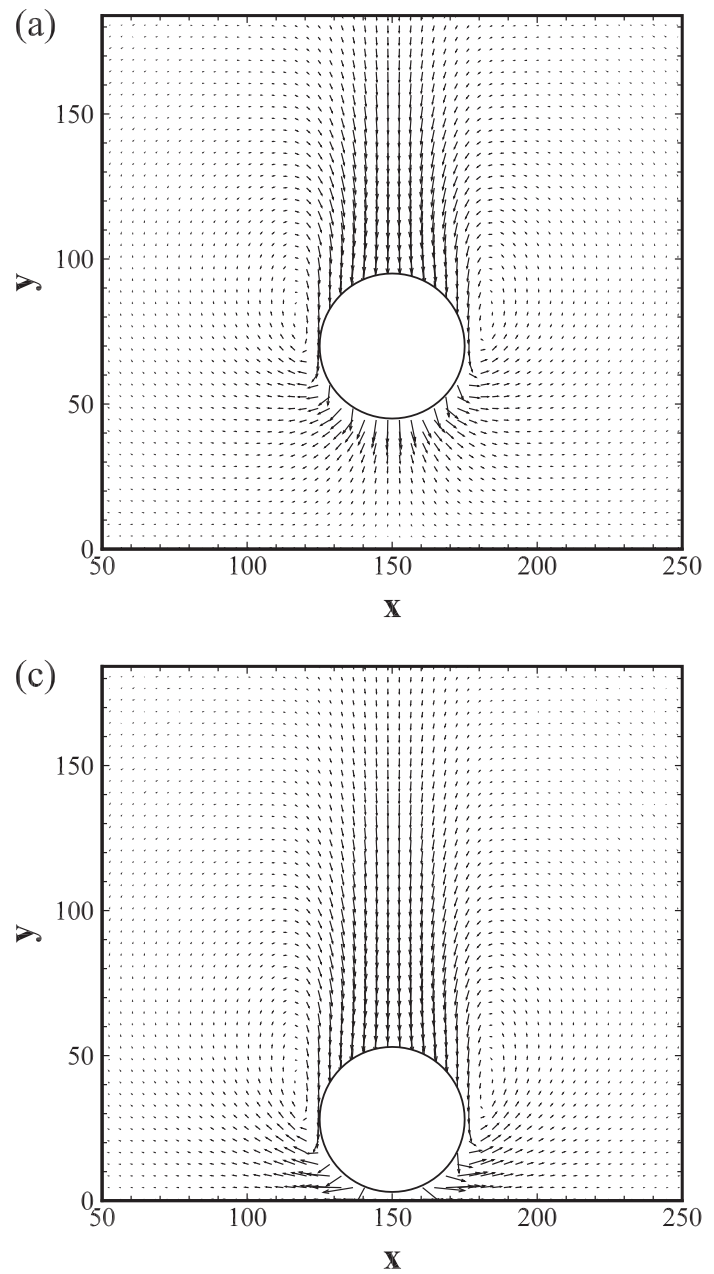
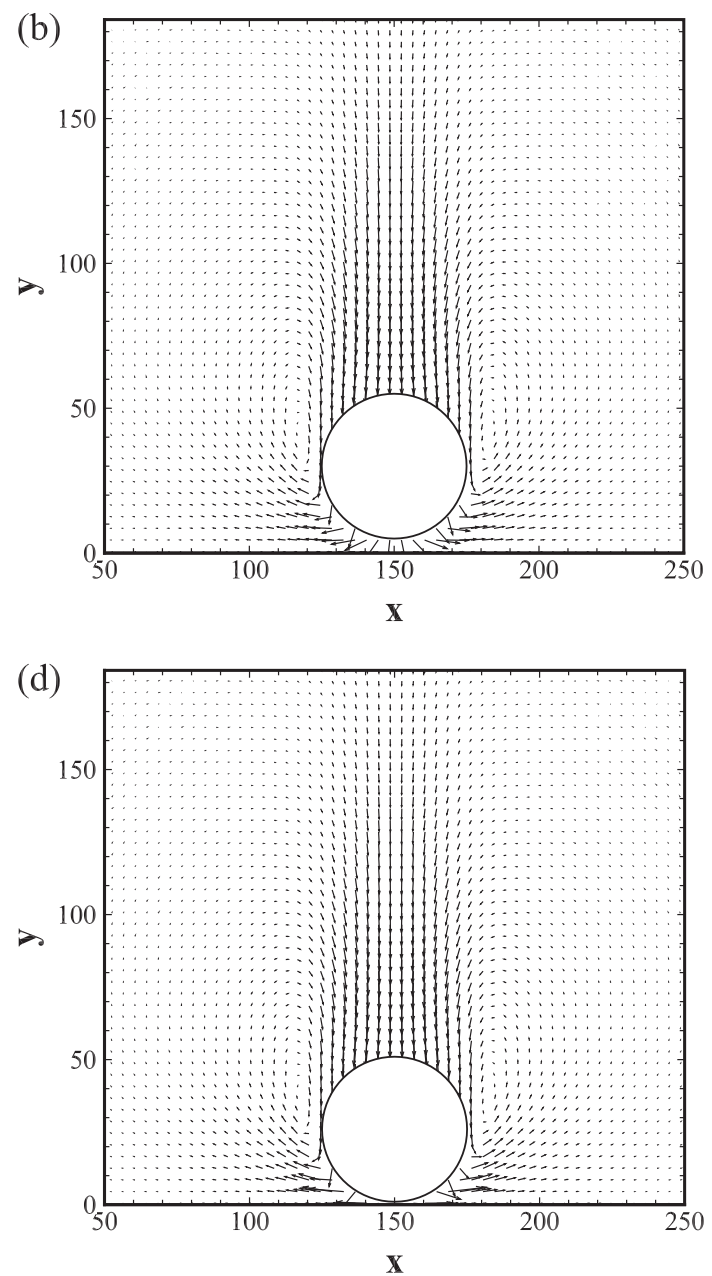

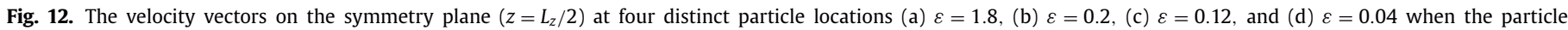
moves normal to the wall in fluid flow at $R e_{p}=140.719$.

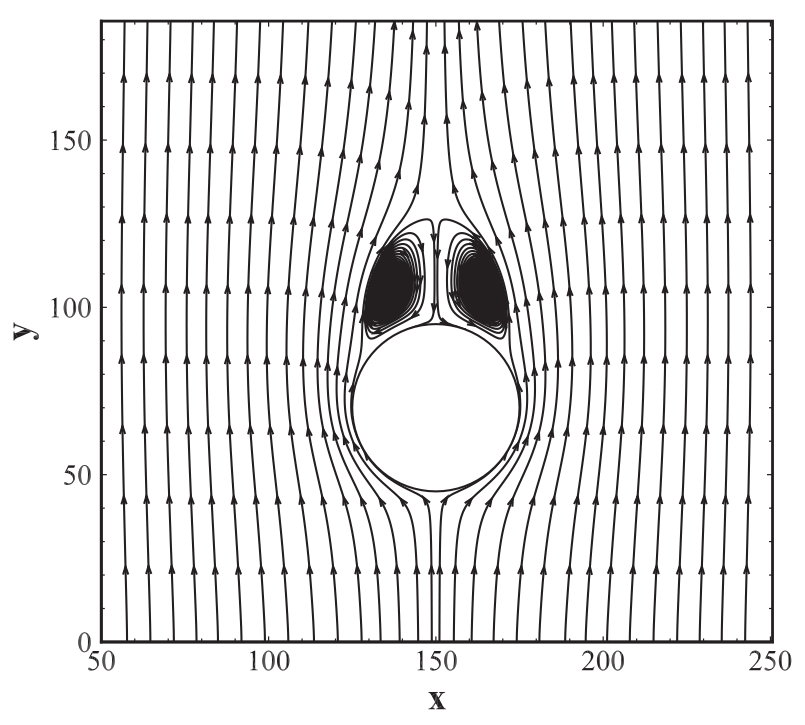

Fig. 13. The streamline obtained from the related velocity $\left(u_{f}-U, w\right)$ plot at $R e_{p}=$ $140.719, u_{f}$ and $U$ are respectively the fluid velocity and particle falling velocity in the negative $y$-direction at $\varepsilon=1.8$. correlated formula to the drag coefficient

$$
\begin{aligned}
A_{11}= & \frac{1}{\varepsilon}-\frac{1}{5} \ln \varepsilon-\frac{1}{21} \varepsilon \ln \varepsilon+\left(0.325+0.207 \sqrt{\operatorname{Re}_{p}}+0.0045 \operatorname{Re}_{p}\right) \\
& +\left(0.22+0.002 \operatorname{Re}_{p}\right),\left(3 \leq \operatorname{Re}_{p} \leq 50\right)
\end{aligned}
$$

Fig. 15 plots the coefficient $A_{11}$ as a function of $\varepsilon$ at $R e_{p}=$ 30.500. There is a small discrepancy between the corrected formula proposed in the present work and that of Lin and Lin (2013). However, the drag coefficient given in Eq. (3) proposed by Cox and Brenner (1967) coincides well with the corrected formula proposed in the present work. It seems that the asymptotic expression based on $\varepsilon \ll 1$ and $\varepsilon R e_{p} \ll 1$ proposed by Cox and Brenner (1967) has a wider application range shown in Fig. 15 than the theoretical limitation $\varepsilon \operatorname{Re}_{p} \ll 1$.

\subsubsection{Particle moving parallel to the wall}

For the other three types of particle motion, the gap between the particle and the wall are set to $0.5,0.75,1.0,1.5,2.0$, and 4.0 at different particle Reynolds numbers. The numerical values of coefficients are denoted as $A_{22}^{N}, B_{23}^{N}, D_{33}^{N}$ and $D_{11}^{N}$ respectively. For different $R e_{p}$ and $\varepsilon$, we can get the models for hydrodynamic force and torque under stationary state. The corrected formula of coefficient $A_{22}$ is expressed as Eq. (32) with $R e_{p}$ and $\varepsilon$. Eq. (33) is derived from Eq. (32). Fig. 16 shows the plots of Eqs. (32) and (33). 

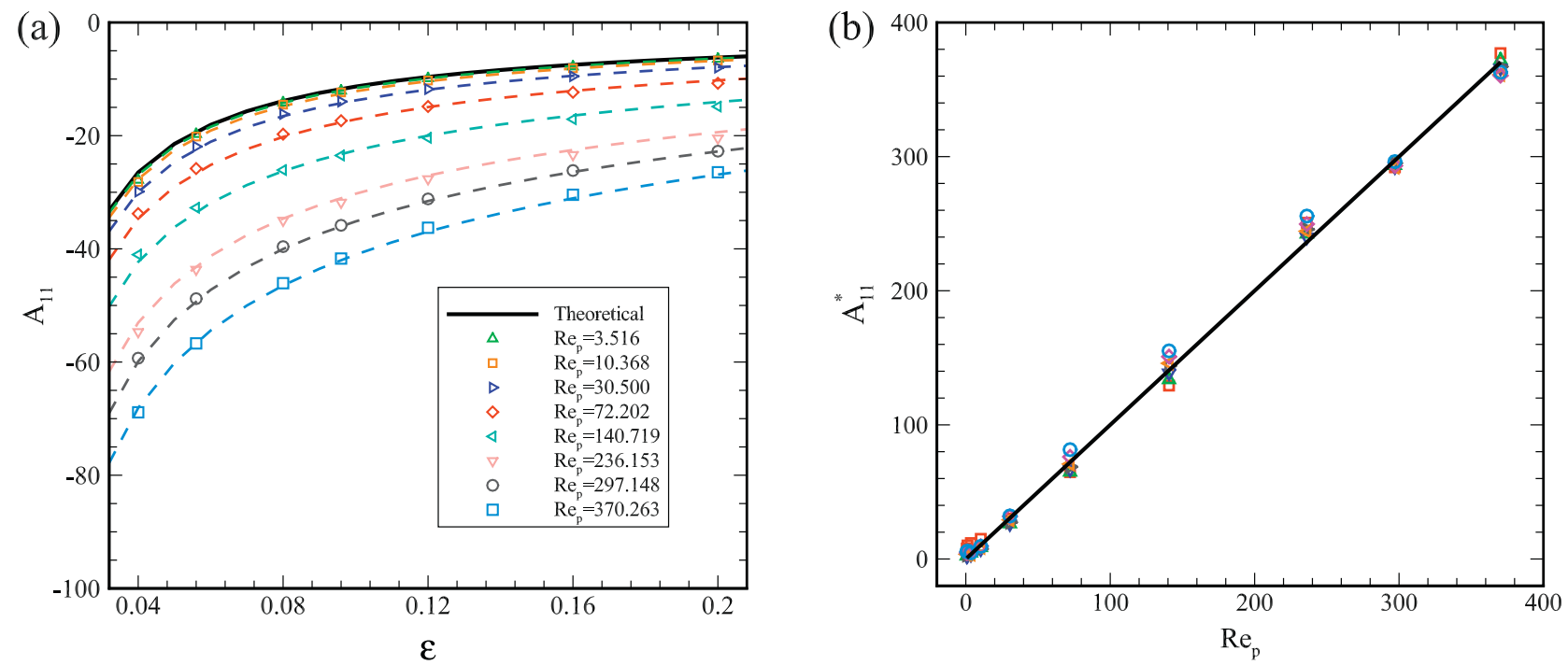

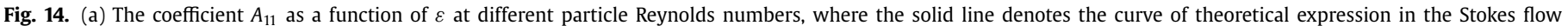

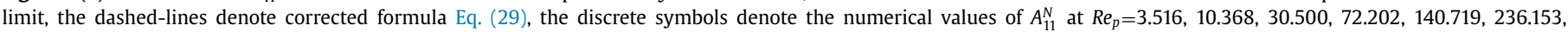

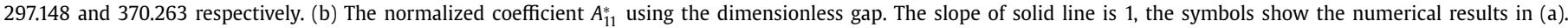

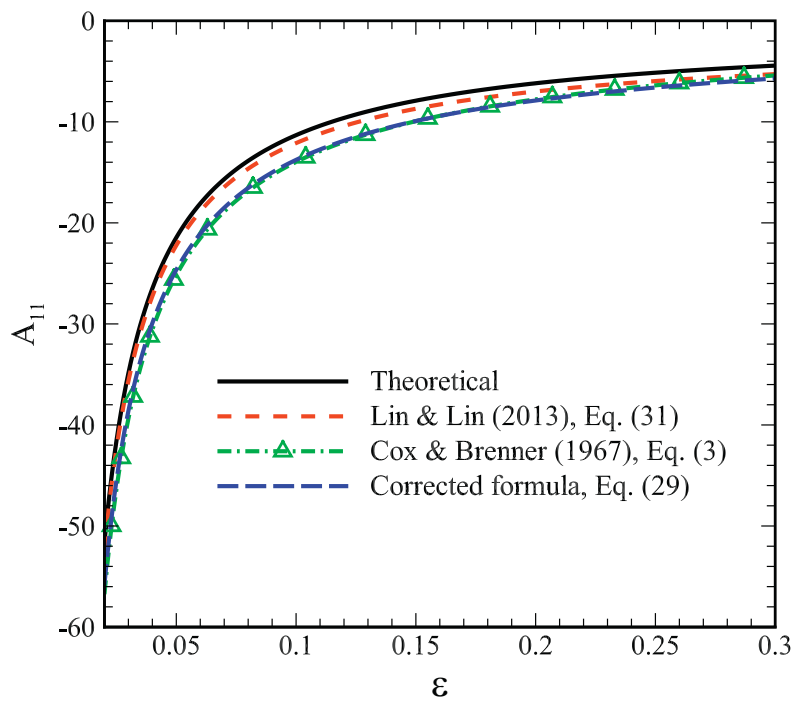

Fig. 15. The coefficient $A_{11}$ as a function of $\varepsilon$ at $R e_{p}=30.500$. The solid line: theoretical expression in the Stokes flow limit; dash line: the corrected formula given in Eq. (31) proposed by Lin and Lin (2013); dash dot line with symbols: Eq. (3) proposed by Cox and Brenner (1967); long dash line: the corrected formula given in Eq. (29) proposed in the present work.

$A_{22}=A_{22}^{T}+(-0.0861+0.0118 \cdot \varepsilon) \cdot R e_{p}^{0.83}, \quad R e_{p} \leq 370$,

$A_{22}^{*}=\frac{A_{22}-A_{22}^{T}}{-0.0861+0.0118 \cdot \varepsilon}=R e_{p}^{0.83}$.

In Fig. 16(a), the curve of corrected formula of $A_{22}$ agrees with the curve of theoretical expression listed in line 3 of Table 2 when $R e_{p}$ vanishes. With the increasing of $R e_{p}$, the absolute value of $A_{22}$ increases for a given $\varepsilon$. For different $R e_{p}$, the numerical values of $A_{22}$ denoted with discrete symbols are all close to the curves of the corrected formula. Similar to Fig. 14(b), the collapse of all lines at different particle Reynolds numbers in Fig. 16(b) confirms that Eq. (32) is capable of capturing the effect of particle Reynolds number on $A_{22}$.

\subsubsection{Particle rotating around $\mathrm{z}$ axis}

In the third type, two coefficients $B_{23}^{N}, D_{33}^{N}$ are simultaneously calculated. The coefficients $B_{23}, D_{33}$ expressed with $\varepsilon$ and $R e_{\Omega}$ are obtained and depicted as Eqs. (34) and (36), respectively. Figs. 17 and 18 show the coefficients $B_{23}, D_{33}$ versus $\varepsilon$ and $R e_{\Omega}$.

$$
\begin{aligned}
& B_{23}=B_{23}^{T}+\left(-0.00246-3.69 \times 10^{-4} \cdot \varepsilon^{-1 / 2}\right) \cdot R e_{\Omega}^{0.83}, \\
& \quad \operatorname{Re}_{\Omega} \leq 236,
\end{aligned}
$$

$B_{23}^{*}=\frac{B_{23}-B_{23}^{T}}{-0.00246-3.69 \times 10^{-4} \cdot \varepsilon^{-1 / 2}}=R e_{\Omega}^{0.83}$.

$$
D_{33}=D_{33}^{T}-\left(0.02195 \cdot e^{-\varepsilon / 0.00924}+0.00254+0.00423 \cdot \varepsilon\right) \cdot \operatorname{Re}_{\Omega},
$$$$
\operatorname{Re}_{\Omega} \leq 370 \text {, }
$$

$$
D_{33}^{*}=\frac{D_{33}-D_{33}^{T}}{-\left(0.02195 \cdot e^{-\varepsilon / 0.00924}+0.00254+0.00423 \cdot \varepsilon\right)}=\operatorname{Re}_{\Omega}
$$

It is observed that the curves of corrected formulae of $B_{23}$ and $D_{33}$ agree with the curves of the theoretical expressions listed in lines 4 and 5 of Table 2 at $\operatorname{Re}_{\Omega} \rightarrow 0$. For a given $\varepsilon$, the absolute value of $D_{33}$ increases with $R e_{\Omega}$ monotonically as shown in Fig. 18(a), but the value of $B_{23}$ decreases with the increasing of $R e_{\Omega}$ in Fig. 17(a). It is very interesting that when $\operatorname{Re}_{\Omega}$ is small, the value of $B_{23}$ is positive, and then it becomes negative with increasing $R e_{\Omega}$. At small rotational particle Reynolds numbers and sphere-wall gap widths, the viscous stresses are large and the rotation of sphere is impeded by the wall, and the force $F_{X}$ is negative. At large rotational particle Reynolds numbers, the pressure gradient that develops along the gap between the sphere surface and the wall dominates over the viscous stress on the sphere surface, which is strong enough to deflect the force $F_{x}$. With the increasing of sphere-wall gap width, the viscous effect becomes less important and the value of $F_{x}$ becomes positive at intermediate rotational particle Reynolds numbers. This argument is carefully analyzed by using the decomposition of the force on the particle as follows.

To figure out why the coefficient $B_{23}$ varies from a positive value to a negative one with increasing $R e_{\Omega}$, we focus upon a value 

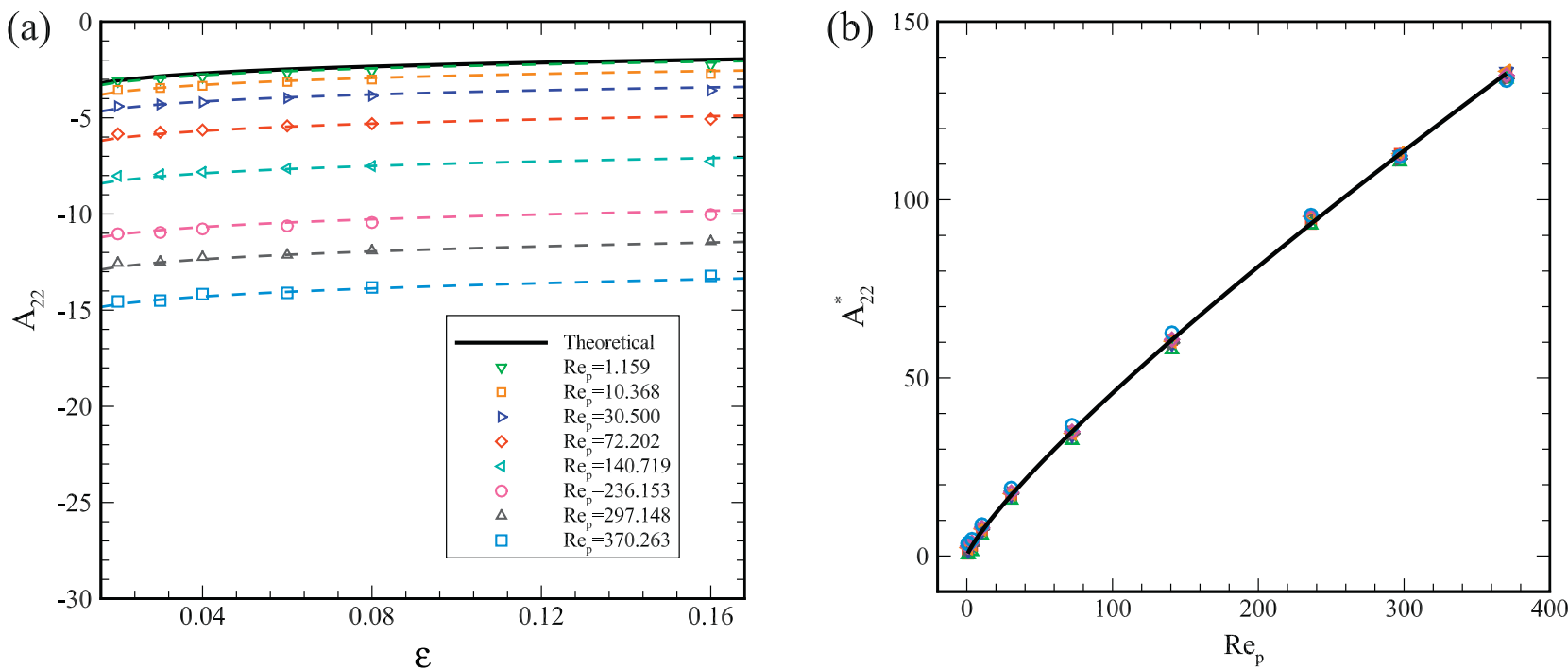

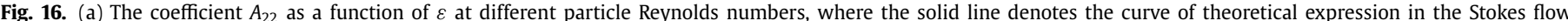

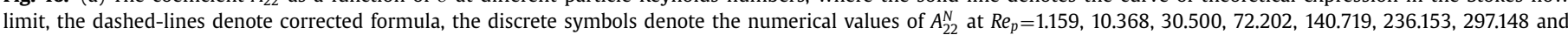

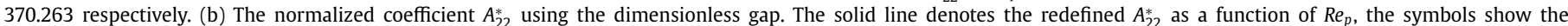
numerical results.
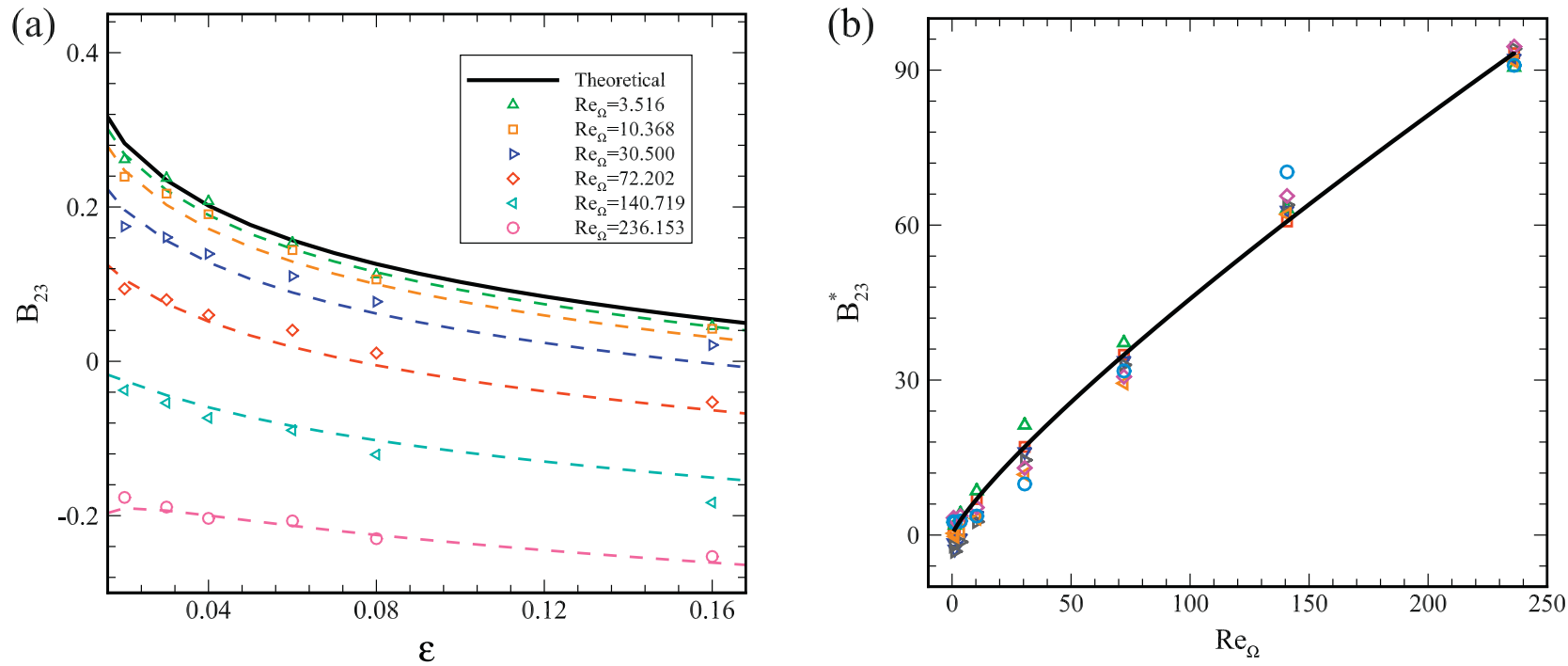

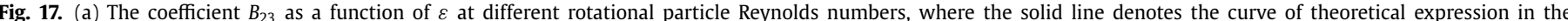

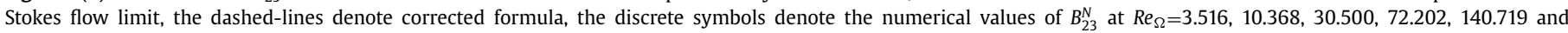

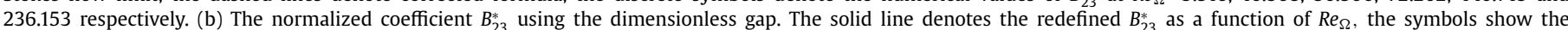
numerical results.

of $\varepsilon=0.16$ and compute the force $F_{X}$ using the velocity and pressure fields by surface integration. Fig. 19 plots the pressure contours on the plane $\left(z=L_{z} / 2\right)$ when the particle rotates around $z$ axis located at $\varepsilon=0.16$ in fluid flow at $\operatorname{Re}_{\Omega}=3.516,10.368,30.500$, $72.202,140.719$ and 236.153 respectively.

In Fig. 19, the contours are asymmetric along $z$ axis and $z=L_{z} / 2$ is the plane of symmetry, the values of pressure on the left side of particle are larger than that on the right side at different $\operatorname{Re}_{\Omega}$. The surface of particle is divided into many small surface elements of spherical coordinate. The pressure integration on the particle can be simply computed using the pressure field. Meanwhile, the viscous stress on each surface element can be computed using velocity gradient field. The viscous force on the particle is obtained using superposition of the product of viscous stress and surface area. The contribution of viscous force and pressure to the coefficient $B_{23}$ on the particle can be obtained as shown in Fig. 20. It is clear that the total value of $B_{23}$ computed by the velocity and pressure fields is close to the value computed using LBM. The coefficient $B_{23}$ varies from a positive value to a negative one mainly due to its dramatic increasing of contribution of pressure.

Lee and Balachandar (2010) have considered the case of a particle rotating around $x$ axis parallel to the wall in quiescent ambient fluid. Note that the rotational particle Reynolds number was defined as $\operatorname{Re}_{\Omega}^{\prime}=|\tilde{\Omega}| d^{2} / \nu=2 R e_{\Omega}$ in their work, with $\tilde{\Omega}$ the angular velocity of particle. The rotational drag and moment coefficients on the particle were defined as follows:

$$
\begin{aligned}
& C_{D \Omega}=\frac{-F_{x}}{\frac{\pi}{32} \rho \tilde{\Omega}|\tilde{\Omega}| d^{4}}=B_{23} \cdot \frac{48}{R e_{\Omega}^{\prime}}, \\
& C_{M \Omega}=\frac{T_{z}}{\frac{\pi}{64} \rho \tilde{\Omega}|\tilde{\Omega}| d^{5}}=-D_{33} \cdot \frac{64}{R e_{\Omega}^{\prime}} .
\end{aligned}
$$



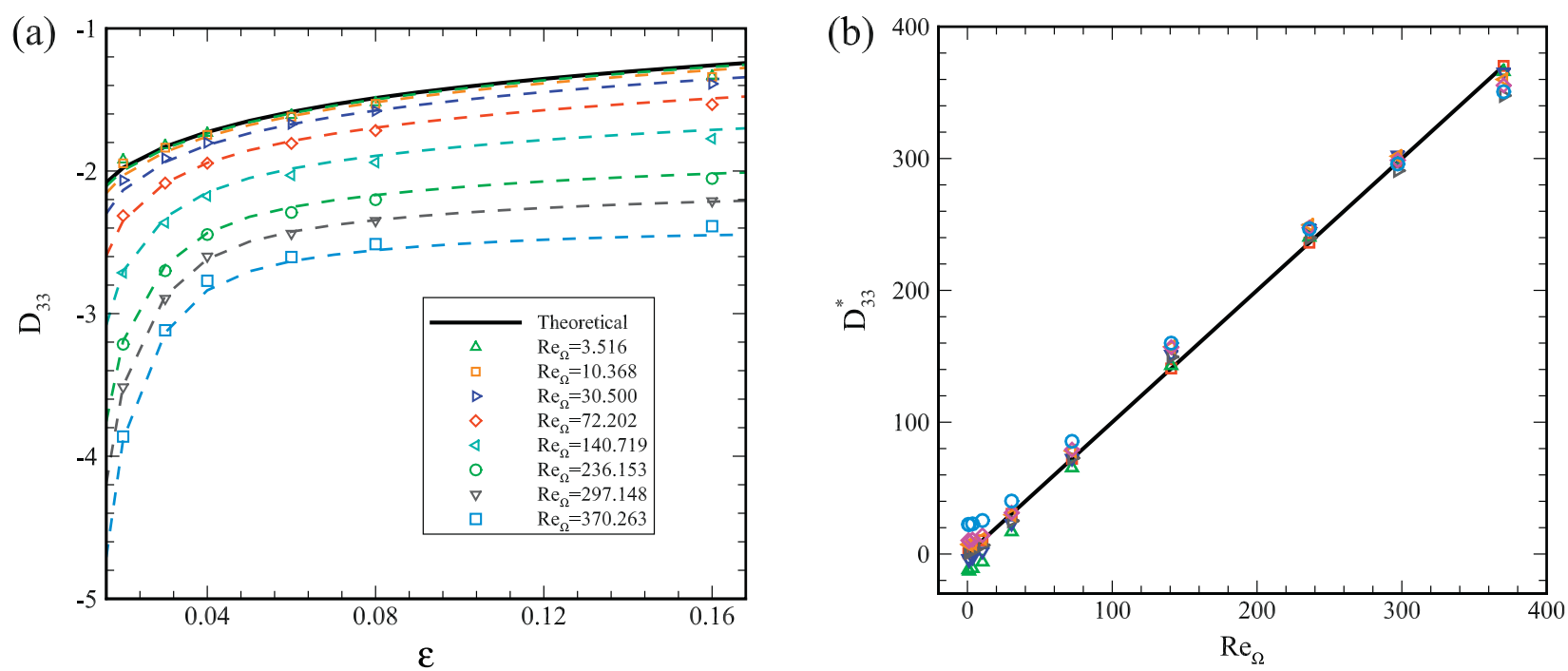

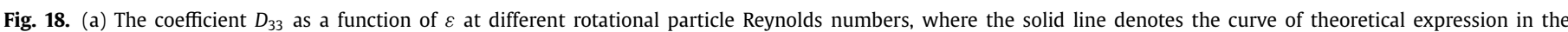

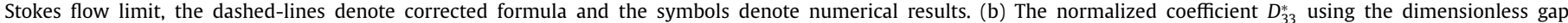
versus particle Reynolds number, calculated using Eq. (37), the symbols show the numerical results.
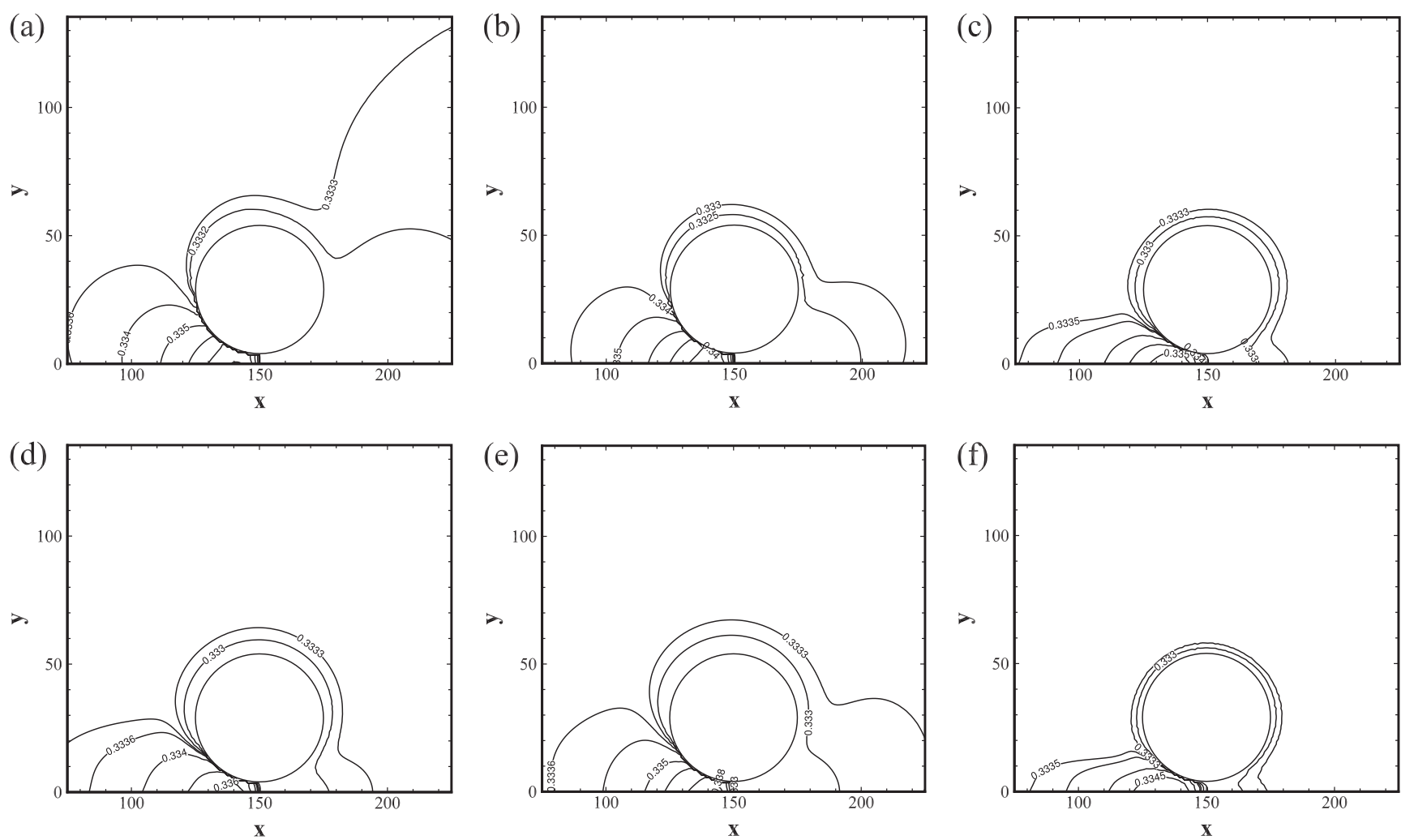

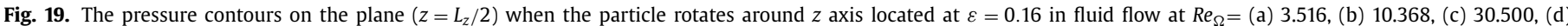
72.202, (e) 140.719 , and (f) 236.153.

Fig. 21 shows the drag and moment coefficients for a rotating particle for different $R e_{\Omega}^{\prime}$ and $\varepsilon$. The current models for $B_{23}$ and $D_{33}$ denoted as thick lines are reasonably consistent with the drag and moment correlations proposed by Lee and Balachandar (2010) denoted as thin lines.

\subsubsection{Particle rotating around y axis}

For the case of particle rotating around $y$ axis, comparison of the discrete coefficient $D_{11}^{N}$ at $\operatorname{Re}_{\Omega} \rightarrow 0$ is made with the theoretical results of coefficient $D_{11}$ from Jeffery (1915). The coefficient
$D_{11}^{N}$ at $R e_{\Omega}=0.6485$ is computed for comparison. It's obvious that the curve of $D_{11}^{N}$ at $R e_{\Omega}=0.6485$ is consistent with Jeffery's result, as depicted in Fig. 22, which validates Jeffery's result using lattice Boltzmann method.

Through data fitting, we get the formula of coefficient $D_{11}^{T}$ of Jeffery's theoretical result for $\varepsilon \leq 0.5$ as

$D_{11}^{T}=-1.277+0.285 \cdot \varepsilon^{0.25}$ 


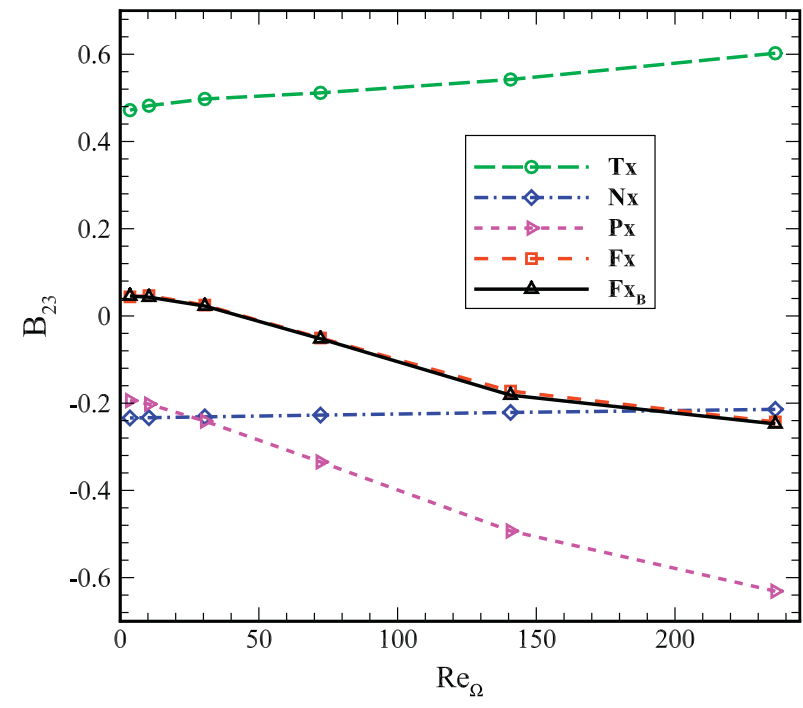

Fig. 20. Comparisons of the contribution of tangential viscous stress $\operatorname{Tx}$ (long dash line), normal viscous stress $\mathrm{Nx}$ (dash dot line) and pressure $\mathrm{Px}$ (short dash line) to the coefficient $B_{23}$ versus $\operatorname{Re}_{\Omega}$. The dash line denoted as "Fx" is the value of $B_{23}$ calculated from the velocity and pressure fields, which is the sum of the above individual contributions to $B_{23}$. The solid line denoted as " $\mathrm{FX}_{\mathrm{B}}$ " is the value of $B_{23}$ for $\varepsilon=0.16$ at different $\operatorname{Re}_{\Omega}$ from Fig. 17(a).

The numerical values of $D_{11}^{N}$ can be used to get the corrected formula expressed with $\varepsilon$ and $R e_{\Omega}$, as shown

$D_{11}=D_{11}^{T}-(0.00329+0.00141 \cdot \varepsilon) \cdot R e_{\Omega}, \quad R e_{\Omega} \leq 370$,

$D_{11}^{*}=\frac{D_{11}-D_{11}^{T}}{-0.00329-0.00141 \cdot \varepsilon}=R e_{\Omega}$.

Fig. 23(a) shows the curves of coefficient $D_{11}$ as a function of $\varepsilon$ at different $\operatorname{Re}_{\Omega}$. For a given $\varepsilon$, the absolute value of coefficient $D_{11}$ increases with $\operatorname{Re}_{\Omega}$ monotonically. In Fig. 23(b), the values of $D_{11}^{*}$ at different $R e_{\Omega}$ are close to the line with a slope of 1 , demonstrating the validity of Eq. (40).

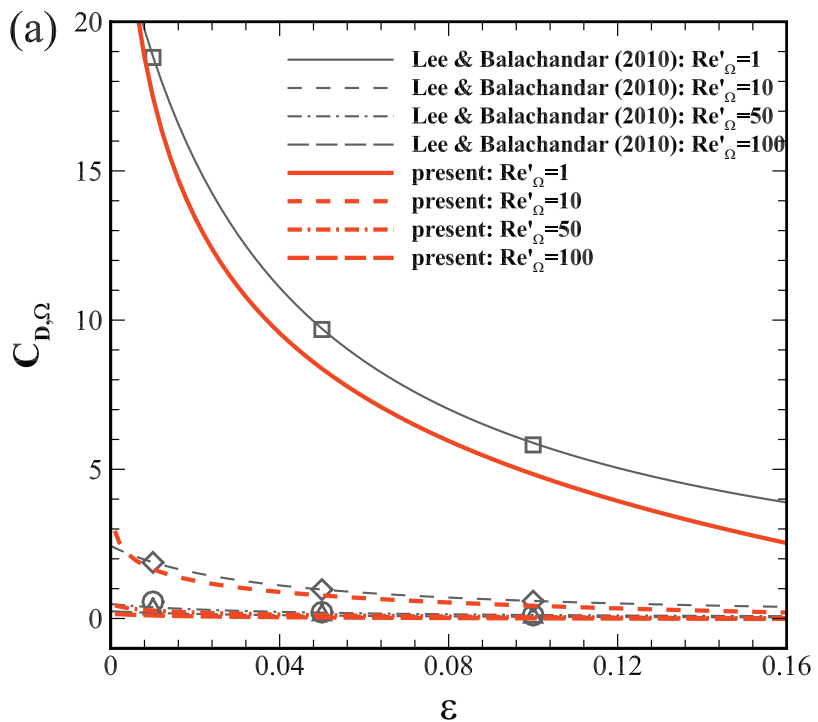

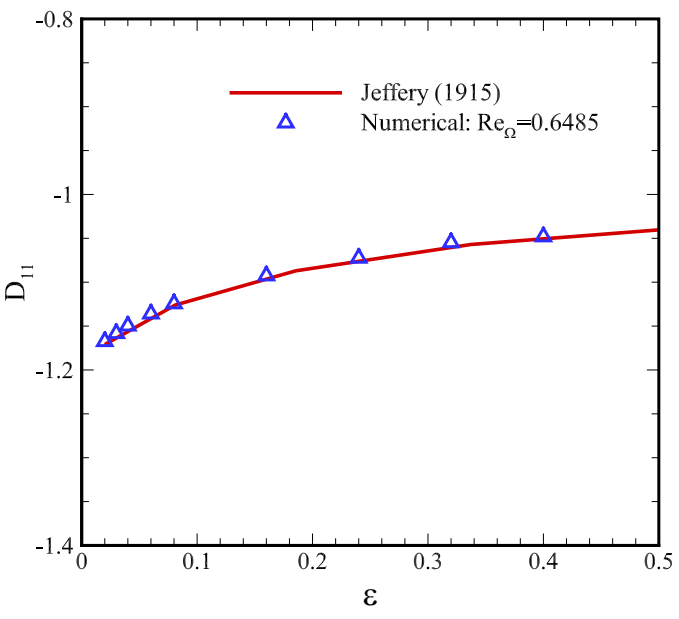

Fig. 22. The numerical values of $D_{11}$ at $R e_{\Omega}=0.6485$ comparing with theoretical results of $D_{11}$ obtained by Jeffery (1915).

\subsection{Validation}

\subsubsection{Particle's general motions}

After obtaining the models for the hydrodynamic force and torque through four simple motions of particle, it is necessary to validate the accuracy of the models through particle's general motions to study the effects of the nonlinear coupling characteristics of fluid flow on particle motions.

For the first example, parameters are set to $R e_{p}=30.500$ and $v_{x}=0.015, v_{y}=0.02$, where $v_{x}, v_{y}$ are respectively velocity of $x$ direction and $y$-direction. This is the particle's composite motion of the first and second types in Section 2.3. The coefficients $A_{11}$ and $A_{22}$ as a function of $\varepsilon$ are both depicted in Fig. 24.

In Figs. 24-26, the dash line and the solid line respectively denote the curves of theoretical expressions in Stokes flow limit and the proposed models. The gradient symbols denote the numerical results. In Fig. 24, it can be observed that the coefficients in particle's composite motion are much closer to the proposed models than the theoretical expressions in the Stokes flow limit,

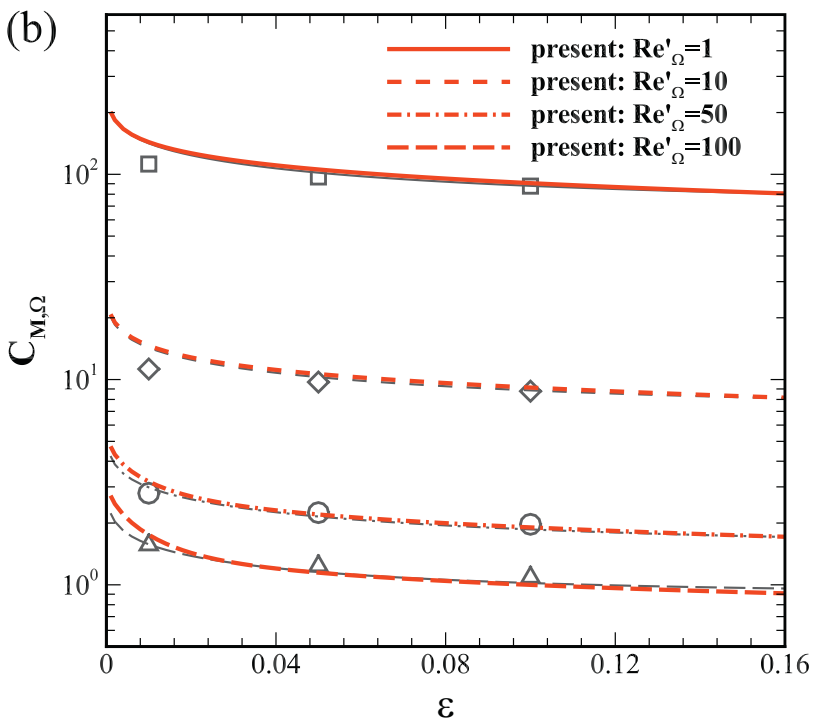

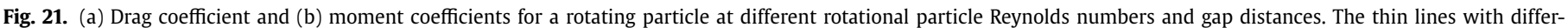

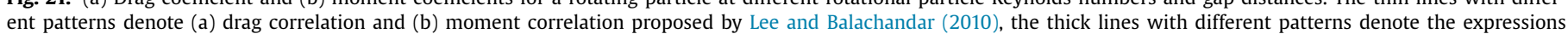
transformed from the corrected formula of (a) $B_{23}$ given in Eq. (34) and (b) $D_{33}$ given in Eq. (36). The symbols denote numerical results from Lee and Balachandar (2010). 
(a)

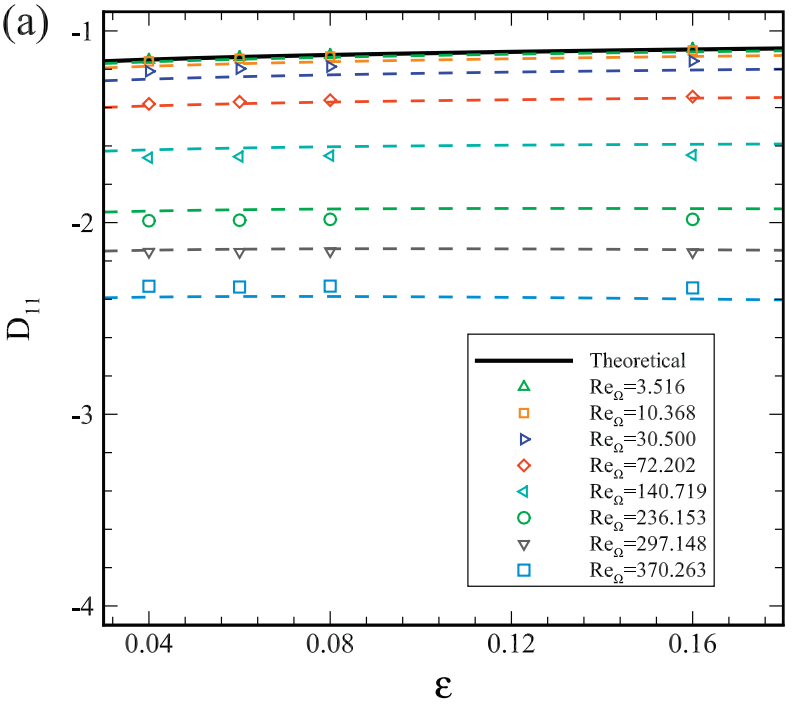

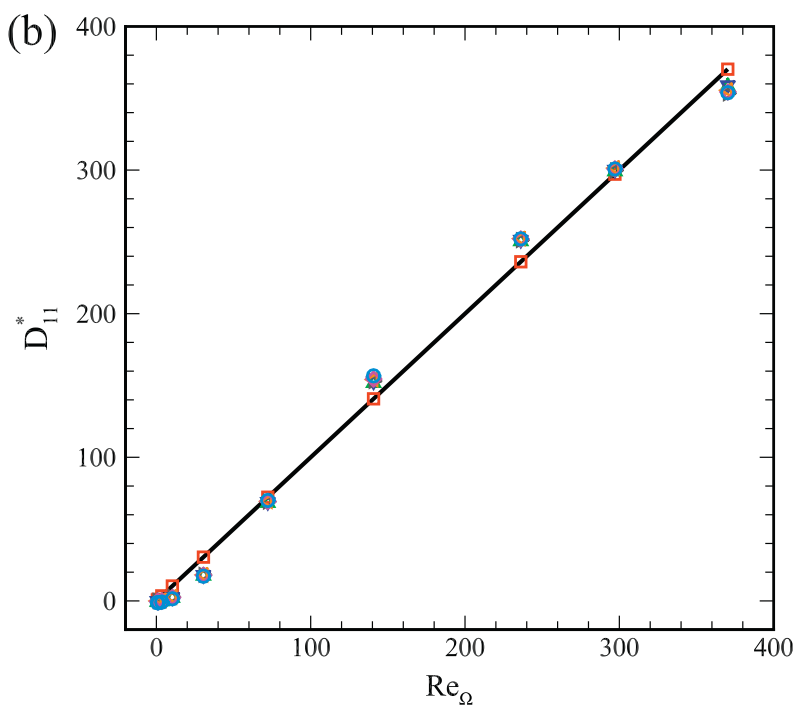

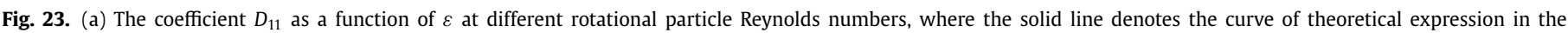

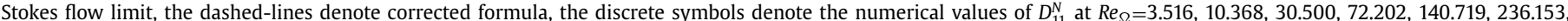
297.148 and 370.263 respectively. (b) The normalized coefficient $D_{11}^{*}$ using the dimensionless gap. The slope of solid line is 1 , the symbols show the numerical results.
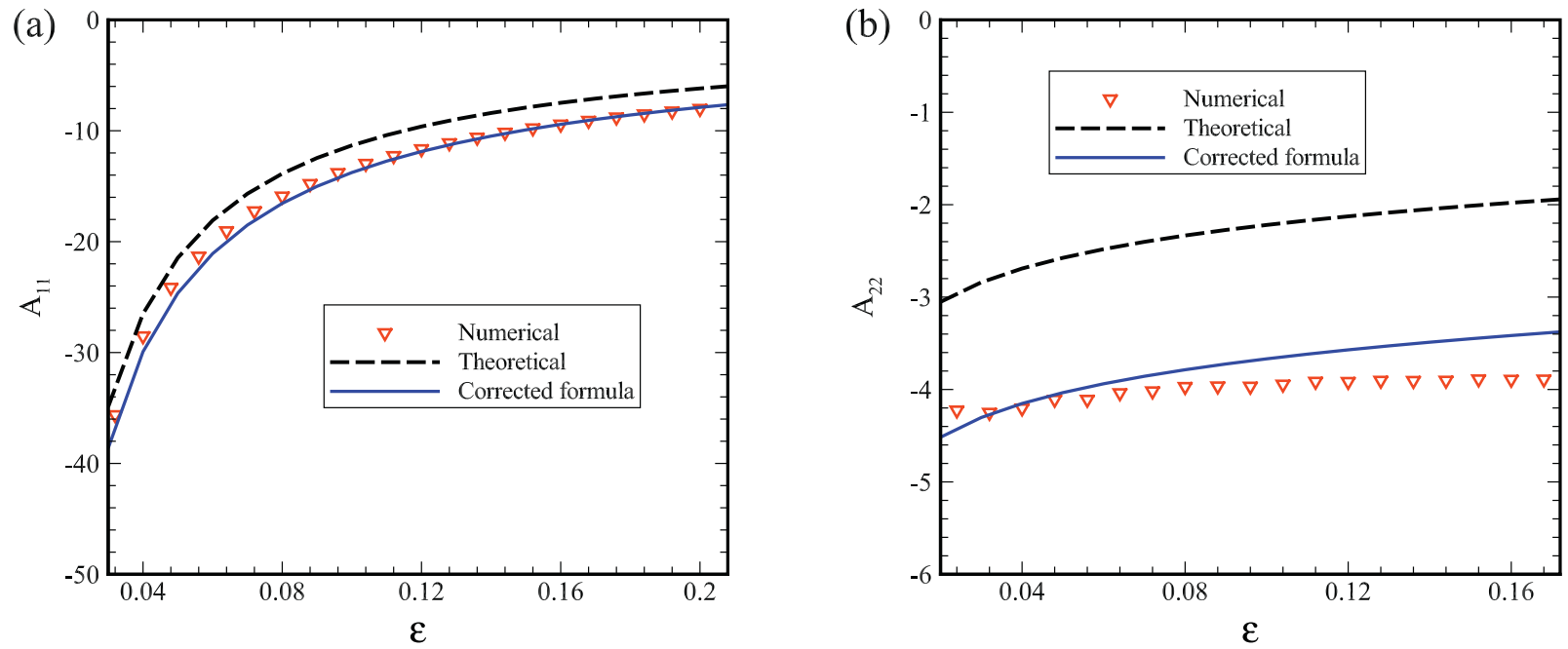

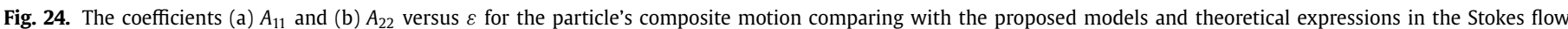
limit when $R e_{p}=30.500$.
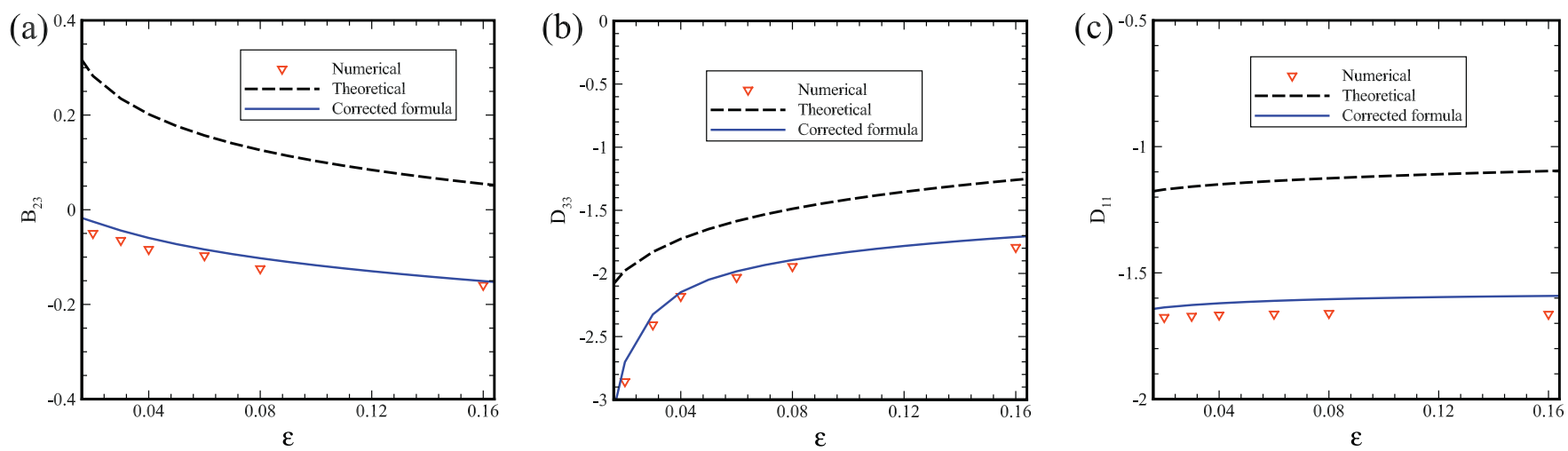

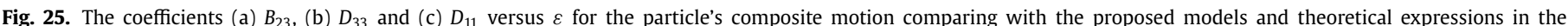
Stokes flow limit when $\operatorname{Re}_{\Omega}=140.719$. 
(a)

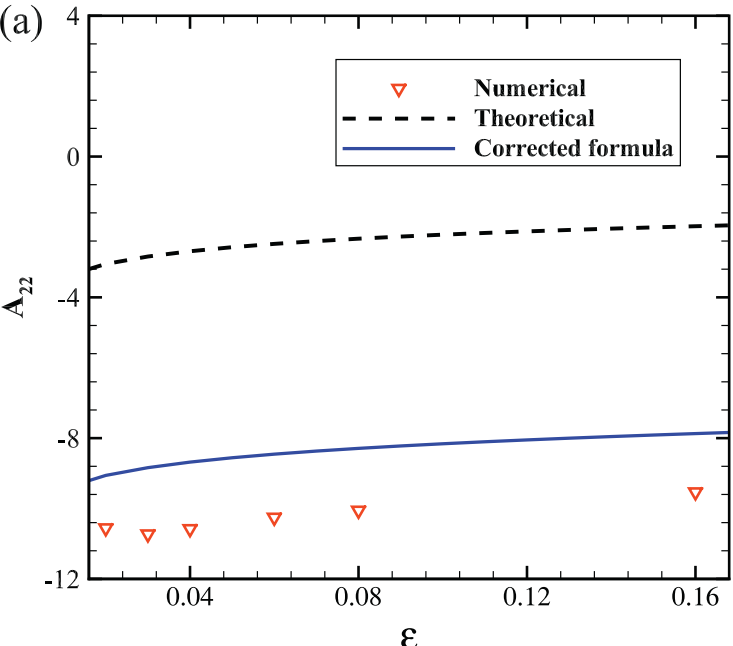

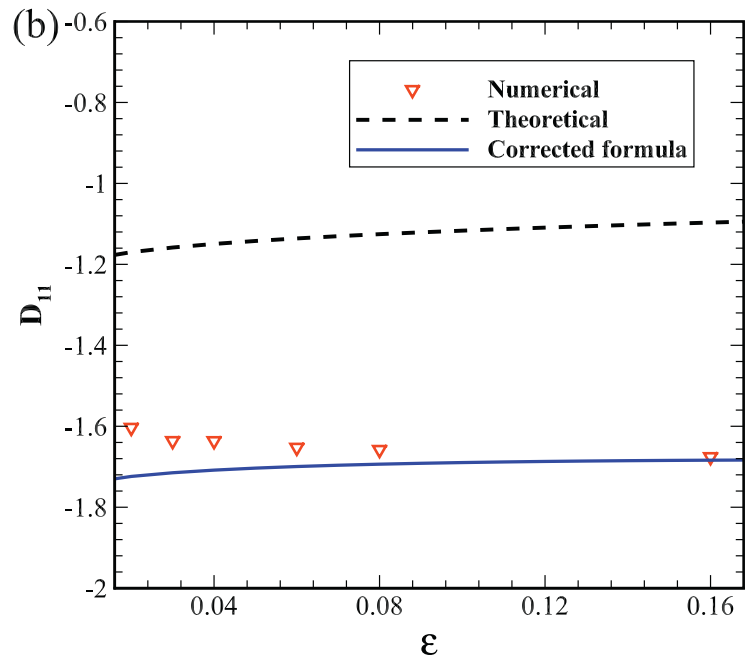

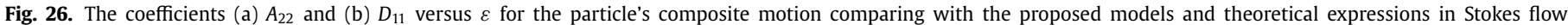
limit when $R e_{p}=R e_{\Omega}=166.986$

which denotes that the corrected formulae can significantly improve the prediction of $A_{11}$ and $A_{22}$ of a particle in a general motion.

For the second example, parameters are set to $R e_{\Omega}=140.719$ and $\Omega_{y}=\Omega_{z}=0.002$, where $\Omega_{y}, \Omega_{z}$ are respectively angular velocity of the particle rotating around $y$ and $z$ axis. This is the particle's composite motion of the third and fourth types in Section 2.3. The plots of coefficients $B_{23}, D_{33}$ and $D_{11}$ versus $\varepsilon$ are depicted in Fig. 25.

Fig. 25 shows that the numerical results of $B_{23}, D_{33}$ and $D_{11}$ for the particle's composite motion coincide well with the proposed models, demonstrating that the proposed models are applicable for the general motion of a particle.

For the third example, parameters are set to $R e_{p}=R e_{\Omega}=$ 166.986, $v_{x}=0.025 * \sqrt{2}$ and $\Omega_{y}=0.001 * \sqrt{2}$, which represents a more general case with both translational and rotational motions. This is the particle's composite motion of the second and fourth types in Section 2.3. The coefficients $A_{22}$ and $D_{11}$ versus $\varepsilon$ are plotted in Fig. 26.

Considering the effects of inherent nonlinearity of the governing equations of the flow at finite particle Reynolds number, we get a reasonable agreement between the proposed models and the numerical data compared with the linear lubrication theory, as shown in Fig. 26.

4.6.2. The validation of proposed models through comparison with previous modeling results

For further validation of the proposed models, we shall compare the corrected formulae with the modeling results by Lee and Balachandar (2010) and Lee et al. (2011). In this case, the hydrodynamic force on a finite-sized particle undergoing both translation along $x$ axis and rotation around $z$ axis in quiescent ambient fluid close to the wall was studied using an immersed boundary technique (Lee and Balachandar, 2010; Lee et al., 2011). According to Lee and Balachandar (2010), the translation Reynolds numbers is defined as $R e_{p}=\left|\tilde{V}_{p}\right| d / v$, with $\tilde{V}_{p}$ the translational velocity of the particle. The translational drag coefficient on the particle is defined as

$C_{D t}=\frac{F_{x}}{\frac{\pi}{8} \rho \tilde{V}_{p}\left|\tilde{V}_{p}\right| d^{2}}=-A_{22} \cdot \frac{24}{R e_{p}}$.

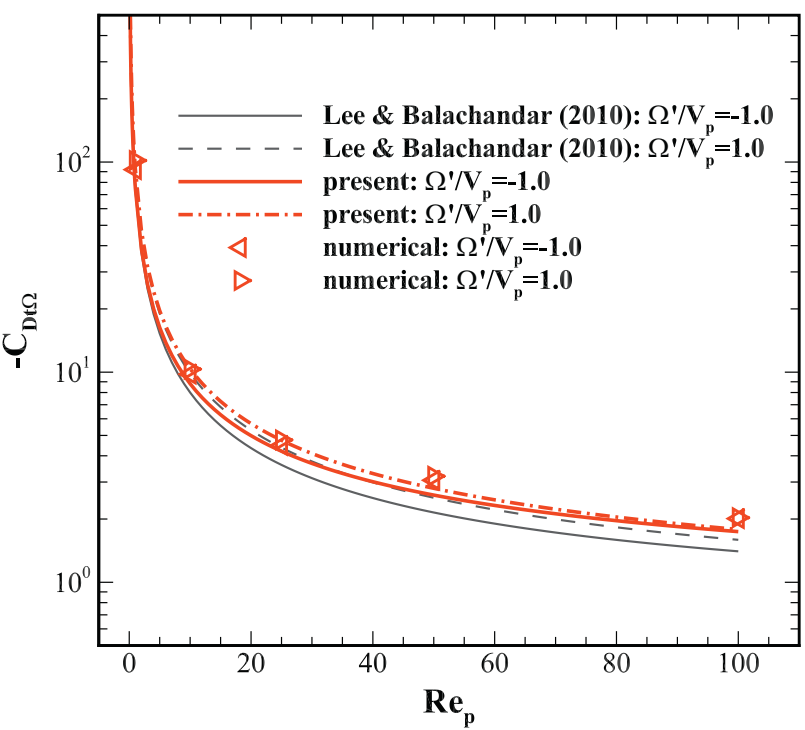

Fig. 27. Drag coefficients of a translating-rotating sphere in a stagnant ambient flow when $L=0.505(\varepsilon=0.01)$. The thin lines denote the drag correlation Eq. (43) proposed by Lee and Balachandar (2010), the thick lines denote the linear superposition of corrected formulae of $A_{22}$ and $B_{23}$ given in Eq. (44) and the numerical results are plotted as symbols.

For a given dimensionless gap $L=0.505$ or $\varepsilon=0.01$, they gave the expressions,

$\left\{\begin{array}{l}C_{D t \Omega}=-C_{D t}-C_{D \Omega} \cdot \frac{\Omega^{\prime}}{V_{p}}\left|\frac{\Omega^{\prime}}{V_{p}}\right|-\frac{\Omega^{\prime}}{V_{p}} g_{t \Omega}, \\ C_{D t}(L=0.505)=\frac{81.96}{R e_{p}}\left(1+0.01 R e_{p}^{0.959}\right), \\ C_{D \Omega}(L=0.505)=\frac{18.84}{R e_{\Omega}^{\prime}} .\end{array}\right.$

In the current model, we can relate the drag coefficient $C_{D t \Omega}$ on a particle with both translational and rotational motions as

$C_{D t \Omega}=A_{22} \cdot \frac{24}{R e_{p}}-B_{23} \cdot \frac{48}{R e_{\Omega}^{\prime}} \cdot \frac{\Omega^{\prime}}{V_{p}}\left|\frac{\Omega^{\prime}}{V_{p}}\right|$.

Fig. 27 shows the drag coefficients plotted against the translational Reynolds number for different ratios of $\Omega^{\prime} / V_{p}$ when $L=0.505$ or $\varepsilon=0.01$. The non-dimensional quantity $\Omega^{\prime} / V_{p}=$ $(\tilde{\Omega} d / 2) / \tilde{V}_{p}$. The thick lines denote the combination of corrected formulae of $A_{22}$ and $B_{23}$ given in Eq. (44), which coincide well with 
the drag correlation Eq. (43) without including translation-rotation coupling term $g_{t \Omega}$ proposed by Lee and Balachandar (2010) and denoted as thin lines. The drag coefficients obtained from the present numerical simulations, which include the translation-rotation coupling effects, are shown with symbols. It can be observed that the translation-rotation coupling effects could be ignored over the parameter range considered and the results obtained from particle's general motion of translation-rotation using the lattice Boltzmann method are validated.

\section{Conclusions}

In this paper, the models for the hydrodynamic force and torque acting on a particle moving near a solid wall in a viscous fluid at finite particle Reynolds numbers are proposed. The conventional lubrication theory for force and torque is based on the assumption of Stokes flow at vanishing particle Reynolds number, which is not suitable for general situations at finite particle Reynolds numbers. Therefore, this paperwork is aimed to consider the effects of finite particle Reynolds numbers on the hydrodynamic force and torque. The multiple-relaxation-time lattice Boltzmann model is used to simulate the flow field induced by the prescribed motion of a finite-size spherical particle moving at a given particle Reynolds number. The method is extensively validated using previous numerical and experimental data. The resolution is up to 50 grids per particle diameter. Given different particle Reynolds numbers and gap sizes between the sphere and the wall, we can compute the coefficients of hydrodynamic force and torque. The results show that the effects of finite particle Reynolds number have a significant impact on the coefficients of hydrodynamic force and torque at a given gap.

We obtain the models for the hydrodynamic force and torque, as shown in Eqs. (29), (32), (34), (36) and (40) respectively. When the particle Reynolds number is close to be zero, the values of coefficients as a function of $\varepsilon$ all approach the conventional lubrication theory. For a given $\varepsilon$, the absolute values of coefficients $A_{11}$, $A_{22}, D_{33}$ and $D_{11}$ increase with particle Reynolds number monotonically, while the coefficient $B_{23}$ varies from a positive value to a negative value. To understand this variation in $B_{23}$, we compute the individual component of forces along the $x$ axis on the particle by integrating the viscous stress and pressure over the particle surface when it rotates around the $z$ axis in the fluid flow at increasing $R e_{\Omega}$. It is discovered that the coefficient $B_{23}$ varies from a positive value to a negative value mainly due to the asymmetric distribution of pressure. The asymmetric distribution of pressure leads to a rapid increase of negative contribution to $B_{23}$.

We further validate the new developed models for the hydrodynamic force and torque, not only by numerically calculating particle's general motions but also by comparing with available models from literature. Those validations demonstrate that the proposed models can be used in general motions in which the fluid motions in different directions induced by the particle moving at finite particle Reynolds number are coupled. The proposed models for the hydrodynamic force and torque can be used as sub-grid models in any PR-DNS simulation approaches where the flow in the gap between the particle and wall can not be sufficiently resolved, and in Lagrangian simulations of particle-laden flows where particles are very close to a wall, such as particles in cyclone separators.

\section{Acknowledgement}

This work was supported by the National Natural Science Foundation of China(11472277 and 11232011), Strategic Priority Research Program, CAS (XDB22040104), Key Research Program of Frontier Sciences, CAS (QYZDJ-SSW-SYS002), Science Challenge Program (JCKY2016212A501), the 973 Program of
China (2013CB834100) and Natural Science Foundation of Shandong Province (ZR2011EL035).The anonymous reviewers are much appreciated for the constructive suggestions to improve the manuscript.

\section{References}

Belt, R.J., Daalmans, A.C.L.M., Portela, L.M., 2012. Experimental study of particledriven secondary flow in turbulent pipe flows. J. Fluid Mech. 709, 1-36. doi:10. 1017/jfm.2012.104.

Bianco, F., Chibbaro, S., Marchioli, C., Salvetti, M.V., Soldati, A., 2012. Intrinsic filtering errors of Lagrangian particle tracking in LES flow fields. Phys. Fluids 24, 045103. doi: $10.1063 / 1.3701378$.

Bouzidi, M., Firdaouss, M., Lallemand, P., 2001. Momentum transfer of a Boltzmannlattice fluid with boundaries. Phys. Fluids 13(11), 3452-3459. doi:10.1063/1. 1399290.

Caiazzo, A., 2008. Analysis of lattice Boltzmann nodes initialisation in moving boundary problems. Prog. Comput. Fluid Dyn. 8, 3-10. doi:10.1504/PCFD.2008. 018074.

Capecelatro, J., Pepiot, P., Desjardins, O., 2014. Numerical characterization and modeling of particle clustering in wall-bounded vertical risers. Chem. Eng. J. 245, 295-310. doi:10.1016/j.cej.2014.02.040.

ten Cate, A., Nieuwstad, C.H., Derksen, J.J., den Akker, H.E.A.V., 2002. Particle imaging velocimetry experiments and lattice-Boltzmann simulations on a single sphere settling under gravity. Phys. Fluids 14(11), 4012-4025. doi:10.1063/1. 1512918.

Clift, R., Grace, J.R., Weber, M.E., 1978. Bubbles, Drops, and Particles. Academic Press.

Cooley, M.D.A., O'Neill, M.E., 1969. On the slow motion generated in a viscous fluid by the approach of a sphere to a plane wall or stationary sphere. Mathematika 16, 37-49. doi:10.1112/S0025579300004599.

Cox, R., Brenner, H., 1967. The slow motion of a sphere through a viscous fluid towards a plane surface-II Small gaps widths, including inertial effects. Chem. Eng. Sci. 22, 1753â-1777. doi:10.1016/0009-2509(67)80208-2.

Dance, S.L., Maxey, M.R., 2003. Incorporation of lubrication effects into the forcecoupling method for particulate two-phase flow. J. Comput. Phys. 189, 212-238. doi:10.1016/S0021-9991(03)00209-2.

d'Humieres, D., Ginzburg, I., Krafczyk, M., Lallemand, P., Luo, L.S., 2002. Multiplerelaxation-time lattice Boltzmann models in three dimensions. Phil. Trans. R. Soc. Lond. A 360, 437-451. doi:10.1098/rsta.2001.0955.

Dorgan, A.J., Loth, E., 2004. Simulation of particles released near the wall in a turbulent boundary layer. Int. J. Multiphase Flow 30, 649-673. doi:10.1016/j. ijmultiphaseflow.2004.05.006.

Gao, H., Li, H., Wang, L.P., 2013. Lattice Boltzmann simulation of turbulent flow laden with finite-size particles. Comp. Math. Appl. 65, 194-210. doi:10.1016/j. camwa.2011.06.028.

García-Villalba, M., Kidanemariam, A.G., Uhlmann, M., 2012. DNS of vertical plane channel flow with finite-size particles: Voronoi analysis, acceleration statistics and particle-conditioned averaging. Int. J. Multiphase Flow 46, 54-74. doi:10. 1016/j.ijmultiphaseflow.2012.05.007.

Gondret, P., Lance, M., Petit, L., 2002. Bouncing motion of spherical particles in fluids. Phys. Fluids 14, 643-652. doi:10.1063/1.1427920.

He, G.W., Rubinstein, R., Wang, L.P., 2002. Effects of subgrid-scale modeling on time correlations in large eddy simulation. Phys. Fluids 14, 2186-2193. doi:10.1063/1. 1483877.

Jeffery, G.B., 1915. On the steady rotation of a solid of revolution in a viscous fluid. Phil. Mag. s2_14(1), 327-338. doi:10.1112/plms/s2_14.1.327.

Jeffrey, D.J., 1982. Low-Reynolds-number flow between converging spheres. Mathematika 29, 58-66. doi:10.1112/S002557930001216X.

Jeffrey, D.J., Onishi, Y., 1984. The forces and couples acting on two nearly touching spheres in low-Reynolds-number flow. J. Appl. Math. Phys. 35, 634-641. doi:10. 1007/BF00952109.

Joseph, G., Zenit, R., Hunt, M., Rosenwinkel, A., 2001. Particle-wall collisions in a viscous fluid. J. Fluid Mech. 433, 329-346. doi:10.1017/S0022112001003470.

Kidanemariam, A.G., Chan-Braun, C., Doychev, T., Uhlmann, M., 2013. Direct numerical simulation of horizontal open channel flow with finite-size, heavy particles at low solid volume fraction. New J. Phys. 15, 025031. doi:10.1088/1367-2630/ $15 / 2 / 025031$.

Kim, J., Balachandar, S., 2012. Mean and fluctuating components of drag and lift forces on an isolated finite-sized particle in turbulence. Theor. Comput. Fluid Dyn. 26, 185-204. doi:10.1007/s00162-010-0219-1.

Kim, S., Karrila, S.J., 2005. Microhydrodynamics: Principles and Selected Applications. Dover Publications.

Ladd, A.J.C., 1994. Numerical simulations of particulate suspensions via a discretized Boltzmann equation. Part 1. Theoretical foundation. J. Fluid Mech. 271, 285-309. doi:10.1017/S0022112094001771.

Ladd, A.J.C., 1994. Numerical simulations of particulate suspensions via a discretized Boltzmann equation. Part 2. Numerical results. J. Fluid Mech. 271, 311-339. doi: $10.1017 /$ S0022112094001783.

Laín, S., Sommerfeld, M., 2012. Numerical calculation of pneumatic conveying in horizontal channels and pipes: Detailed analysis of conveying behaviour. Int. J. Multiphase Flow 39, 105-120. doi:10.1016/j.ijmultiphaseflow.2011.09.006.

Lallemand, P., Luo, L.S., 2003. Lattice Boltzmann method for moving boundaries. J. Comput. Phys. 184, 406-421. doi:10.1016/S0021-9991(02)00022-0. 
Lee, H., Balachandar, S., 2010. Drag and lift forces on a spherical particle moving on a wall in a shear flow at finite Re. J. Fluid Mech. 657, 89-125. doi:10.1017/ S0022112010001382.

Lee, H., Ha, M.Y., Balachandar, S., 2011. Rolling/sliding of a particle on a flat wall in a linear shear flow at finite Re. Int. J. Multiphase Flow 37, 108-124. doi:10.1016/ j.ijmultiphaseflow.2010.10.005.

Lin, S.Y., Lin, J.-F., 2013. Numerical investigation of lubrication force on a spherical particle moving to a plane wall at finite Reynolds numbers. Int. J. Multiphase Flow 53, 40-53. doi:10.1016/j.ijmultiphaseflow.2013.01.006.

Liu, D., Keaveny, E.E., Maxey, M.R., Karniadakis, G.E., 2009. Force-coupling method for flows with ellipsoidal particles. J. Comput. Phys. 228, 3559-3581. doi:10. 1016/j.jcp.2009.01.020.

Liu, Q.L., Prosperetti, A., 2010. Wall effects on a rotating sphere. J. Fluid Mech. 657, 1-21. doi:10.1017/S002211201000128X.

Lu, H., Chen, J.H., Liu, G.D., Lu, H.L., Li, D., Zhao, F.X., 2013. Simulated second-order moments of clusters and dispersed particles in riser. Chem. Eng. Sci. 101, 800812. doi:10.1016/j.ces.2013.06.036.

Lucci, F., Ferrante, A., Elghobashi, S., 2010. Modulation of isotropic turbulence by particles of Taylor length-scale size. J. Fluid Mech. 650, 5-55. doi:10.1017/ S0022112009994022.

Nguyen, N.Q., Ladd, A.J.C., 2002. Lubrication corrections for lattice-Boltzmann simulations of particle suspensions. Phys. Rev. E 66, 046708. doi:10.1103/PhysRevE. 66.046708 .

O'Neill, M.E., Majumdar, S.R., 1970. Asymmetrical slow viscous fluid motions caused by the translation or rotation of two spheres. Part I: the determination of exact solutions for any values of the ratio of the radii and separation parameters. J. Appl. Math. Phys. 21, 164-179. doi:10.1007/BF01590641.

Ozdemir, C.E., HSU, T.J., Balachandar, S., 2010. A numerical investigation of fine particle laden flow in an oscillatory channel: the role of particle-induced density stratification. J. Fluid Mech. 665, 1-45. doi:10.1017/S0022112010003769.

Portela, L.M., Oliemans, R.V.A., 2003. Eulerian-Lagrangian DNS/LES of particleturbulence interactions in wall-bounded flows. Int. J. Numer. Meth. Fluids 43, 1045-1065. doi:10.1002/fld.616.

Qi, D.W., He, G.W., Liu, Y.M., 2014. Lattice Boltzmann simulations of a pitch-up and pitch-down maneuver of a chord-wise flexible wing in a free stream flow. Phys. Fluids 26, 021902. doi:10.1063/1.4866182.

Rong, X.Y., Qi, D.W., He, G.W., Zhu, J.Y., Scott, T., 2008. Single curved fiber sedimentation under gravity. Comput. Math. Appl. 55, 1560â1567. doi:10.1016/j.camwa. 2007.08.014.

Rosa, B., Wang, L.P., Maxey, M.R., Grabowski, W.W., 2011. An accurate and efficient method for treating aerodynamic interactions of cloud droplets. J. Comput. Phys. 230, 8109-8133. doi:10.1016/j.jcp.2011.07.012.

Shao, X.M., Wu, T.H., Yu, Z.S., 2012. Fully resolved numerical simulation of particleladen turbulent flow in a horizontal channel at a low Reynolds number. J. Fluid Mech. 693, 319-344. doi:10.1017/jfm.2011.533.

Song, C.M., Pei, B.B., Jiang, M.T., Wang, B., Xu, D.L., Chen, Y.X., 2016. Numerical analysis of forces exerted on particles in cyclone separators. Powder Technol. 294, 437-448. doi:10.1016/j.powtec.2016.02.052.

Tagawa, Y., van der Molen, J., van Wijngaarden, L., Sun, C., 2013. Wall forces on a sphere in a rotating liquid-filled cylinder. Phys. Fluids 25, 063302. doi:10.1063/ 1.4811406 .

Tenneti, S., Subramaniam, S., 2014. Particle-resolved direct numerical simulation for gas-solid flow model development. Annu. Rev. Fluid Mech. 46, 199-230. doi:10. 1146/annurev-fluid-010313-141344.
Uhlmann, M., 2008. Interface-resolved direct numerical simulation of vertical particulate channel flow in the turbulent regime. Phys. Fluids 20, 053305. doi:10. $1063 / 1.2912459$

Uhlmann, M., Doychev, T., 2014. Sedimentation of a dilute suspension of rigid spheres at intermediate Galileo numbers: the effect of clustering upon the particle motion. J. Fluid Mech. 752, 310-348. doi:10.1017/jfm.2014.330.

Wang, L.M., Zhang, B., Wang, X.W., Ge, W., Li, J.H., 2013. Lattice Boltzmann based discrete simulation for gas-solid fluidization. Chem. Eng. Sci. 101, 228-239. doi:10.1016/j.ces.2013.06.019.

Wang, L.M., Zhou, G.F., Wang, X.W., Xiong, Q.G., Ge, W., 2010. Direct numerical simulation of particle-fluid systems by combining time-driven hard-sphere model and lattice Boltzmann method. Particuology 8, 379-382. doi:10.1016/j.partic. 2010.07.003.

Wang, L.P., Ayala, O., Gao, H., Andersen, C., Mathews, K.L., 2014. Study of forced turbulence and its modulation by finite-size solid particles using the lattice Boltzmann approach. Comput. Math. Appl. 67, 363-380. doi:10.1016/j.camwa.2013.04. 001.

Wang, Z.L., Fan, J.R., Luo, K., 2008. Combined multi-direct forcing and immersed boundary method for simulating flows with moving particles. Int. J. Multiphase Flow 34, 283-302. doi:10.1016/j.ijmultiphaseflow.2007.10.004.

Wu, T.-H., Guo, R.-S., He, G.W., Liu, Y.M., Qi, D.W., 2014. Simulation of swimming of a flexible filament using the generalized lattice-spring lattice-Boltzmann method. J. Theor. Biol. 349, 1-11. doi:10.1016/j.jtbi.2014.01.021.

Xu, H.T., Bodenschatz, E., 2008. Motion of inertial particles with size larger than Kolmogorov scale in turbulent flows. Physica D 237, 2095-2100. doi:10.1016/j. physd.2008.04.022

Yang, Y., He, G.W., Wang, L.P., 2008. Effects of subgrid-scale modeling on Lagrangian statistics in large eddy simulation. J. Turbulence 9, 1-24. doi:10.1080/ 14685240801905360.

Yeo, K., Dong, S., Climent, E., Maxey, M.R., 2010. Modulation of homogeneous turbulence seeded with finite size bubbles or particles. Int. J. Multiphase Flow 36, 221-233. doi:10.1016/j.ijmultiphaseflow.2009.11.001.

Zeng, L.-Y., Balachandar, S., Fischer, P., 2005. Wall-induced forces on a rigid sphere at finite Reynolds number. J. Fluid Mech. 536, 1-25. doi:10.1017/ S0022112005004738.

Zeng, L.Y., Balachandar, S., Fischer, P., Najjar, F., 2008. Interactions of a stationary finite-sized particle with wall turbulence. J. Fluid Mech. 594, 271-305. doi:10. 1017/S0022112007009056.

Zeng, L.Y., Balachandar, S., Najjar, F.M., 2010. Wake response of a stationary finitesized particle in a turbulent channel flow. Int. J. Multiphase Flow 36, 406-422. doi:10.1016/j.ijmultiphaseflow.2010.01.001.

Zhang, W.B., Noda, R., Horio, M., 2005. Evaluation of lubrication force on colliding particles for DEM simulation of fluidized beds. Powder Technol. 158, 92-101. doi:10.1016/j.powtec.2005.04.021.

Zhao, X., He, G.W., 2009. Space-time correlations of fluctuating velocities in turbulent shear flows. Phys. Rev. E 79, 046316. doi:10.1103/PhysRevE.79.046316.

Zhu, L.D., He, G.W., Wang, S.Z., Miller, L., Zhang, X., You, Q., Fang, S.F., 2011. An immersed boundary method based on the lattice Boltzmann approach in three dimensions, with application. Comput. Math. Appl. 61, 3506â3518. doi:10.1016/ j.camwa.2010.03.022 